\title{
Alcance y limitaciones del tratamiento farmacológico del Trastorno por Déficit de Atención e Hiperactividad en niños y adolescentes y Guías de Práctica Clínica. Una revisión bibliográfica.
}

\author{
Outreach and limitations of the pharmacological treatment of Attention Deficit Disorder \\ with Hyperactivity (ADHD) in children and adolescents and Clinical Practice Guidelines. \\ A literature review.
}

\begin{abstract}
Miguel A. Valverde Eizaguirre a , José A. Inchauspe Aróstegui b ${ }^{\text {. }}$
${ }^{a}$ Psicólogo Clínico, Unidad de Hospitalización Psiquiátrica Sección A. Complejo Hospitalario de Navarra. Servicio Navarro de Salud - Osasunbidea. Pamplona, España. ${ }^{b}$ Psiquiatra, Director del Centro de Salud Mental de San Juan. Servicio Navarro de Salud - Osasunbidea. Pamplona, España.
\end{abstract}

Correspondencia: Miguel A. Valverde (mvalvere@cfnavarra.es)

Recibido: 22/02/2013; aceptado: 22/06/2013

\begin{abstract}
RESUMEN: El Trastorno por Déficit de Atención e Hiperactividad (TDA/H) es descrito y presentado en las Guías de Práctica Clínica (GPC) como frecuente en la infancia y adolescencia, asociado a disfuncionalidad significativa en diversos ámbitos, y cuyo tratamiento requiere usar medicamentos, considerados como seguros y efectivos a largo plazo. Se trata de sustancias estimulantes de cuyo daño, en otro tipo de usos en adultos, se ha alertado. En el presente trabajo se ha procedido a una revisión bibliográfica exhaustiva acerca de las evidencias respecto a su efectividad a corto y largo plazo, sobre la sintomatología nuclear del TDA/H, sus resultados funcionales y los riesgos asociados a sus potenciales efectos adversos. El resultado es desalentador y la utilización de estos medicamentos debería considerarse un último recurso, para un número reducido de casos y en periodos de tiempo limitados y breves. PALABRAS CLAVE: Revisión Sistemática, Trastorno por Déficit de Atención e Hiperactividad (TDA/H), Guías de Práctica Clínica (GPC), Metilfenidato, Atomoxetina, Medicina Basada en la Evidencia, Efectividad, Efectos Adversos.
\end{abstract}

ABSTRACT: The usual story about Attention Deficit Hyperactivity Disorder (ADHD), as stated now currently in Clinical Practice Guidelines (CPGs) and passed on to parents and patients, presents ADHD as a common disorder in childhood and adolescence, with very significant dysfunctional consequences in various areas, and whose main therapeutic approach is based on the use of stimulant drugs considered safe and effective in the long term. These drugs are the same stimulants substances whose harmful consequences are well known in other uses in adults. In this paper we have carried out an exhaustive review of the sources from scientific evidence regarding the short and long term effectiveness of the medication, both over the ADHD nuclear symptoms as functional outcomes and risks arising from potential adverse effects. The result is disappointing and should lead to a modification of the CPGs to the use of drugs as tools of last resort, in a small number of cases and limited and short periods of time.

KEY WORDS: Systematic Review, Attention Deficit Hyperactivity Disorder (ADHD), Clinical Practice Guidelines (CPGs), Methylphenidate, Atomoxetine, Evidence-based Medicine, Effectiveness, Adverse Effects. 


\section{Introducción}

El trastorno por déficit de atención con o sin hiperactividad (TDA/H) incluye signos y síntomas en tres áreas consideradas nucleares: impulsividad, atención y movimiento. Aunque se puedan considerar 13 subtipos (1) o incluso 18, según los síntomas de los sistemas diagnósticos ICD-10 y DSM-IV (2), habitualmente se distinguen los subtipos inatento, hipercinético y combinado (2-5).

El TDA/H se relaciona con disfuncionalidad emocional y conductual, y se le atribuyen diversas dificultades en el ámbito familiar, social y escolar del niño y más tarde en aspectos ocupacionales, de búsqueda de empleo, fracaso laboral, accidentabilidad e inserción sociofamiliar (3).

En los setenta, la hiperactividad era un problema infrecuente que podía afectar al $0,002 \%$ de los menores, dos de cada cien mil (6) y hoy, el TDA/H es el diagnóstico más habitual en Salud Mental Infantil, con una prevalencia estimada muy variable, según criterios diagnósticos (se consideran más restrictivos los del ICD10 que los del DSM-IV) países, edad, género (mucho más frecuente en niños que en niñas), clases sociales y grupos étnicos y culturales, entre el 3\% y el 5\% (2-3), pudiendo alcanzar dimensiones epidémicas, entre el 5\% y 10\%, e incluso hasta el $17,8 \%$ (4) y el 19,8\% (3) en determinados grupos y países (7-8).

El incremento de diagnósticos TDA/H, que también se ha dado en nuestro país (5, 9-11), se explica por la evolución de los criterios diagnósticos DSM, cada vez mas laxos $(7,12)$ y otros aspectos propios de la sociología de la medicina, como cambios socioculturales, campañas de detección en el medio escolar y el empuje de la industria farmacéutica (13-14), puesto que la corriente clínica dominante, fuera y dentro de España, propone como abordaje primero y principal del TDA/H el uso de psicofármacos, medicamentos estimulantes y otros de perfil similar (metilfenidato, anfetaminas, atomoxetina) $(5,9,15-18)$. Según estos autores y publicaciones, los psicofármacos ofrecen una alternativa eficaz, mantenida en el tiempo, superior a otros abordajes no farmacológicos y con escasos riesgos a corto y largo plazo, recomendando su toma diaria durante largos periodos de tiempo.

En consecuencia, junto con la dimensión epidémica del diagnóstico de TDA/H, se ha multiplicado el uso de psicofármacos en niños y adolescentes $(19,10,2)$, disminuyendo cada vez más la edad de inicio del tratamiento (2).

En la psicofarmacología del TDA/H se utilizan sustancias estimulantes cuyo daño ha sido reconocido en el uso recreativo en adultos (20). No es extraño que la extensión de su uso en niños y jóvenes, cuyo sistema nervioso está en formación, se haya convertido en un tema de interés y preocupación desde un punto de vista de Salud Pública $(2,10)$, preocupación que se puede rastrear con facilidad en la lectura de Guías de Práctica Clínica, (GPC), aunque sean abiertamente favorables, con matices, al uso de psicofármacos $(3,4,21)$. Por otra parte, se trata de sustancias 
ORIGINALES Y REVISIONES

con potencial de abuso bien conocido y su consumo adictivo parece aumentar paralelamente al de su prescripción (22).

\section{Motivo y objetivos de esta Revisión Bibliográfica}

La literatura científica actual recoge que el $80 \%$ de niños y jóvenes con TDA/H tratados con metilfenidato, el estimulante más utilizado internacionalmente, mejora significativamente $(3,23)$. Si se le resta el efecto placebo, bien conocido en el TDA/H (24), se llegaría a cifras ligeramente menores, en torno al 77\% (2). No obstante, las revisiones sistemáticas se hacen eco de la escasa duración, pobre calidad y los sesgos que caracterizan muchos, si no la mayoría, de estos estudios y sus metaanálisis $(25,26,2)$. Además de los efectos secundarios más comunes, hiporexia, insomnio, cefaleas, etc. se han detectado posibles efectos adversos graves, como sintomatología psicótica y trastornos afectivos (3\% al 6\%), disminución del crecimiento en términos de peso y talla y muerte súbita (2). Y tras "cincuenta años de utilización del metilfenidato en TDA/H” (3) aún parece necesaria la realización de un nuevo metaanálisis de ECAs (estudios controlados y aleatorizados) para valorar el riesgo-beneficio del metilfenidato en niños y jóvenes (2).

La perspectiva científica crítica a la corriente mayoritaria estima que los estudios a largo plazo no muestran dato alguno favorable a los medicamentos y sí indicios de que su uso puede ser contraproducente (27-33). Da por probada la repercusión negativa sobre la talla y el peso y se pregunta sobre su significado respecto al desarrollo madurativo del niño, emitiendo hipótesis inquietantes basadas en modelos animales y estudios in vitro sobre aspectos hormonales, de desarrollo de la pubertad y de neurotoxicidad (27,34-37).

El lector observará que partidarios y detractores comparten, a menudo, las mismas fuentes de información, y en particular para el metilfenidato el estudio $\mathrm{Mul}$ timodal Treatment of Attention Deficit Hyperactivity Disorder (MTA), financiado por el National Institute of Mental Health (NIMH) de USA, y es especialmente manifiesto para las GPC.

Las GPC divergen sobre aspectos esenciales para la práctica clínica a la hora de facilitar orientaciones para iniciar un tratamiento, en qué casos, cuál escoger, durante cuánto tiempo, cuándo interrumpirlo, basándose en qué criterios... o se muestran ambiguas. No se olvide que las propias GPC son valoradas mediante instrumentos ad-hoc (10) que como el AGREE II (38) consideran aspectos formales de las GPC y no evalúan la calidad de los contenidos ni la evidencia sobre la que se sustentan; para ello es necesario acudir a las fuentes citadas cuya calidad sí puede ser evaluada (para los ECA, p.ej., escala de Jadad) (39). 
El clínico, los padres y el niño/adolescente necesitan referencias más sólidas respecto al riesgo/beneficio de la utilización de psicofármacos en el TDA/H y este es el objeto de esta revisión bibliográfica, centrándonos en el metilfenidato y la atomoxetina, únicos fármacos con indicación para el tratamiento del TDA/H en España.

\section{Metodología de la revisión bibliográfica}

Partiendo de la Guía Ministerial Española (5) hemos realizado una lectura detallada de las GPC en las que esta se inspira (3-4) revisando todas las fuentes citadas en todos los aspectos controvertidos, fundamentalmente, los estudios a largo plazo respecto al metilfenidato, MTA a 14 meses (23), a 24 meses (40) y a 3 años $(28-29,41)$ y otros estudios a largo plazo $(42-46)$. Para la atomoxetina se han revisado dos estudios a largo plazo (47-48).

Se han revisado GPCs, publicadas posteriormente, de países representativos, las americanas de la Agency for Healthcare Research and Quality (49), la American Academy of Pediatrics (50) y la australiana NHMRC (21).

Se han revisado metaanálisis, revisiones bibliográficas y estudios comparativos, en particular los de Schachter et al. (51) y King et al. (25) y el estudio de la Universidad de Oregon (26).

Se han buscado, utilizando las bases Medline y PubMed, estudios a largo plazo del uso de metilfenidato y atomoxetina no recogidos por las GPC, revisando para el metilfenidato los resultados del MTA a los 6 y 8 años $(30,31)$ y el estudio australiano RAINE 2011 (33). Para la atomoxetina se ha revisado un estudio de la farmacéutica Eli Lilly en fase III y IV (52).

Se han examinado publicaciones de expertos en TDA/H, incluyendo los críticos a la corriente mayoritaria $(7,20,27,34-35,53-58)$.

No se han revisado aspectos asociados a procedimientos diagnósticos y su validez, epidémicos, neurobiológicos y genéticos, a excepción del mecanismo de acción atribuido a los fármacos, el fenómeno de neuroadaptación, y la naturaleza de los cambios atencionales obtenidos con fármacos, por considerarlos claves a la hora de la prescripción.

\section{Los resultados del tratamiento farmacológico del TDA/H}

Consideraremos separadamente el metilfenidato y la atomoxetina. Dividiremos las pruebas sobre los fármacos a corto y largo plazo, considerando estos últimos a partir de una duración superior a los 12 meses. 
ORIGINALES Y REVISIONES

\subsection{Sobre el metilfenidato}

El metilfenidato es un psicoestimulante. Inhibidor no selectivo de la recaptación de la noradrenalina y, sobre todo, de la dopamina, aumenta la acción de ambos en el córtex prefrontal y en otras áreas cerebrales (59). Está catalogado por la Anatomical Therapeutic Chemical Classification System de la OMS como un simpaticomimético de acción central junto con las anfetaminas y otros medicamentos (60). Su uso y posesión están sujetos a normas regulatorias en diversos países -USA, UK, Canadá, Nueva Zelanda- por su potencial de abuso.

\subsubsection{El metilfenidato a corto plazo}

El metilfenidato se muestra más eficaz que el placebo a corto plazo. Un metaanálisis, asociado a Cochrane Child Health Field, encontró 62 estudios ECA, desde 1981 a 1999, la mitad no pasaban de 10 días, uno duró 28 semanas y nueve más de 4 semanas. El estudio concluye que el metilfenidato tiene un efecto clínico estadísticamente significativo en el tratamiento a corto plazo en individuos de 18 años o menos con diagnóstico de TDA/H. Añade que la superioridad del metilfenidato respecto al placebo en estudios controlados y aleatorizados "más allá de 4 semanas de tratamiento no ha sido demostrado"(51).

Abikoff et al. encuentran que "los preescolares con TDA/H tratados con metilfenidato durante 4 semanas muestran algunas mejoras en el funcionamiento, aunque no tan extensas como las halladas en primaria" (61).

El metaanálisis de la GPC NICE recoge 49 estudios de los que sólo 18 incluyen una comparación con placebo o pacientes en lista de espera, de una duración entre 7 y 192 días, considerando que se muestra más efectivo que el placebo (3).

No nos detendremos en este apartado y daremos por hecho la superioridad de metilfenidato sobre el placebo a corto plazo, al menos hasta 4 semanas.

\subsubsection{El metilfenidato a largo plazo}

No hemos encontrado ECAs de duración superior a seis meses (192 días). Para obtener evidencias sobre lo que ocurre a largo plazo es necesario acudir a estudios de diferente naturaleza, no controlados y aleatorizados, básicamente el MTA, referencia clave sobre el que las diferentes GPCs (3-5), iniciativas de consenso como la Global $\operatorname{ADHD}(17)$ y la mayoría de autores, incluyendo españoles (62), basan sus recomendaciones sobre el uso del metilfenidato, y al más reciente estudio RAINE (33).

Existen otros estudios prolongados hasta cinco años que no son estudios de eficacia y no comparan trayectorias de medicados y no medicados.

Lambert (43-44) examina retrospectivamente a 492 adultos que participaban en un estudio longitudinal, y que habían sido diagnosticados con TDA en los años setenta. Encontró que tenían el doble de uso de tabaco y cocaína los tratados con fármacos que los que no lo fueron. 
Se puede incluir como estudio a largo plazo el de Gillberg (42) pero en el mismo incluye un porcentaje importante de niños autistas y con retraso mental.

Charach et al. siguen durante cinco años a 79 niños que participaron antes en un estudio previo de un año. Observó grandes diferencias en la adherencia al tratamiento y concluye que obtienen mejores resultados quienes cumplen mejor la toma del fármaco, pero afirma que siguen mostrando problemas de TDA/H a los cinco años. Fueron evaluados una vez por año, y se entiende como niño medicado el que ha tomado el fármaco durante dos meses en un año; con este criterio sólo 25 permanecían tomando la medicación a los cinco años. Los niños con medicación mostraban efectos secundarios (45).

Barbaressi et al. realizaron un estudio retrospectivo. En un distrito escolar buscó a niños que fueron diagnosticados de TDA/H, identificando a 378 entre 5718 niños. Eran niños que estudiaron entre 1976-1982 en el distrito y el $77.8 \%$ fueron medicados. Afirma que los niños tomaron fármacos de forma irregular, pero que cuando reiniciaban la toma por tener problemas, se observaron efectos positivos. El estudio no puede comparar la evolución de los medicados y no medicados (46).

El MTA -Multimodal Treatment of Attention Deficit Hyperactivity Disorderfue financiado por el NIMH de USA y contó con la colaboración de reconocidos expertos, parte de ellos con lazos con la industria farmacéutica. El MTA ha generado la publicación de unos setenta artículos (31), más otros que son críticos con el estudio, constituyendo un complejo cuerpo de datos. Los últimos informes sobre el MTA fueron publicados en 2009 y dan cuenta del seguimiento a los ocho años.

\subsubsection{El estudio MTA a los 14 meses (23)}

El MTA es un ensayo multicéntrico que incluyó a 579 menores de 7 a 10 años seleccionados de un grupo de 4541 niños diagnosticados de TDA/H sin que se hayan explicado los criterios de elección. Fueron asignados al azar en cuatro grupos, con una modalidad de tratamiento distinta:

1) Sólo medicación, metilfenidato como primera opción, con una intensa atención clínica a padres y menores, mayor a la habitual.

2) Sólo tratamiento conductual. Se entrenó a padres, menores y profesores. Los profesores recibieron de diez a dieciséis sesiones quincenales. Un supervisor acudió al colegio para ayudar con cada niño durante 12 semanas. Los padres recibieron ayuda en grupo, con un máximo de 27 sesiones, y 8 sesiones individuales. Los niños recibieron terapia en un campamento de verano de 8 semanas que incluyó entrenamiento conductual, con habilidades académicas, sociales, y juegos.

3) Grupo en la comunidad. Niños con TDH/A que siguieron el tratamiento convencional de la época. Dos tercios de sus integrantes tomaban metilfenidato, en dosis menores al grupo 1 y 4, 2 o 3 veces al día. No se informa si algún niño estuvo en tratamiento psicológico.

4) Combinado. Medicación más tratamiento conductual. Recibieron el mismo entrenamiento conductual que el grupo 2 , además de tratamiento farmacológico. 
ORIGINALES Y REVISIONES

El estudio adolece de diversos sesgos que limitan el valor de sus resultados $(63,34)$.

Se realizó mientras se desarrollaba una campaña de sensibilización hacia padres y profesores sobre el TDA/H y el uso del metilfenidato.

Un tercio de los niños tomaba metilfenidato antes del estudio, por lo que una parte de los asignados a sólo terapia conductual sufrieron un proceso de discontinuación farmacológica que posteriormente no se tuvo en cuenta.

Hubo mal cumplimiento en cada grupo. En el grupo de sólo medicación el $22 \%$ no cumplió, en el grupo combinado, el $19 \%$ con la medicación y el $36 \%$ con la terapia conductual, y en el grupo de sólo tratamiento conductual no cumplió el $37 \%$.

No hubo un grupo control comparativo, es decir, un conjunto de niños con TDA/H sin tratamiento alguno. Los autores del estudio lo justifican porque era éticamente inaceptable para un estudio tan largo no utilizar "un tratamiento de reconocida eficacia como la medicación" que la mayoría de pacientes en tratamiento convencional ya estaba tomando (23).

El tratamiento farmacológico y la atención clínica correspondiente se mantuvieron con la misma intensidad de contactos hasta los 14 meses, mientras que el conductual finalizó a los 6-9 meses, diminuyendo luego progresivamente los contactos que pasaron a ser mensuales en los últimos 3-6 meses Se realizó la evaluación al mes del último contacto.

El tratamiento conductual no incluyó componente cognitivo alguno y no era el procedimiento psicólogico más eficaz y eficiente disponible en la época (63).

El estudio no fue cegado. Tanto el alumno como los padres, profesores e investigadores sabían quién tomaba el metilfenidato y quién no. Los niños fueron evaluados por los padres, investigadores, maestros, y los propios menores, y también hubo una evaluación realizada por alguien que desconocía quién tomaba o no el estimulante, mediante observación en el aula.

Buena parte de estos sesgos apuntan a favorecer los grupos con medicación.

El primer informe se realizó a los 14 meses del ensayo y mostró lo siguiente $(7,23,27,34,54,63)$.

a) Que mejoraron considerablemente todos los grupos.

b) Que las diferencias entre grupos fueron pequeñas.

c) Que tuvieron mejores puntuaciones los grupos con medicación que el grupo de terapia conductual y que el grupo en la comunidad.

d) El grupo de medicación se mostró significativamente superior al de terapia conductual en 3 mediciones de un total de 19. Según los profesores la mejoría sólo se dio en atención; según los padres la mejoría sólo se dio en atención y movimiento. En el resto de las 16 mediciones, que incluían ha- 
bilidades sociales, relación padre e hijo, rendimiento escolar, y agresividad, no se halló diferencia en ninguna.

e) El grupo de terapia combinada se mostró superior al de terapia conductual en 5 de 19 mediciones. Inatención según padres y profesores e hiperactividad según padres, así como en conductas agresivas según padres y habilidades lectoras.

f) La evaluación realizada por los niños no indica diferencia alguna: no tenían conciencia subjetiva de mejoría.

g) El $75 \%$ de los niños con sólo terapia conductual se mantuvieron exitosamente durante los 14 meses.

h) El grupo en la comunidad, con dos tercios tomando metilfenidato, no fue mejor que el grupo de terapia conductual.

i) El $34 \%$ de los menores tuvieron problemas de ansiedad y se manejó más eficazmente en el grupo con terapia conductual.

j) El $64 \%$ de los menores que tomaron el fármaco tuvieron efectos secundarios. En el 11,4\% fueron moderados y 2,9\% severos aunque quizás no hubo una observación adecuada para recoger los efectos adversos (63).

k) Las puntuaciones obtenidas mediante observación en el aula por quien desconocía si los niños tomaban o no fármacos no mostró diferencias entre los grupos.

1) En un informe complementario sobre los dos grupos con terapia conductual, se mostró que para el aprendizaje de habilidades en terapia conductual la medicación no era de ayuda (64).

Estos resultados pueden interpretarse entendiendo que los niños con terapia conductual y sin fármacos obtuvieron resultados muy similares a los medicados. No obstante, los investigadores publicitaron que el tratamiento combinado y el medicamentoso se mostraron clínica y estadísticamente superiores en los síntomas TDA/H. Además, argumentaron que, aunque por razones estadísticas las diferencias no fueran significativas, desde un punto de vista clínico sí podrían serlo, haciendo hincapié en que de las 19 variables, el tratamiento combinado se mostró "en cabeza" de los demás en 12, el medicamentoso en 4, el conductual en 2 y el comunitario sólo en 1 (23).

\subsubsection{El MTA a los 24 meses}

Un nuevo informe publicó los resultados a los 2 años (40). A los 14 meses finalizó el ensayo. Se dejó que los alumnos y familiares eligieran el tratamiento que deseaban, y el estudio se transformó en uno de seguimiento prospectivo de una cohorte. De los 579 niños, 540 fueron reexaminados en cinco áreas: a) Síntomas ADHD puntuados por padres y profesores; b) Síntomas de trastorno de oposición desafiante puntuados por padres y profesores; c) Habilidades sociales puntuadas 
ORIGINALES Y REVISIONES

por padres y profesores; d) Habilidades lectoras medidas con el Wechsler Individual Achievement Test Reading; e) La escala Negative Parental Discipline Score.

Las puntuaciones obtenidas en a), c) y e) de quienes recibían medicación empeoraron respecto a las que obtuvieron a los 14 meses, mientras los que estuvieron en el grupo de terapia conductual y en el grupo en la comunidad las mantuvieron.

Los autores concluyeron indicando que los beneficios del fármaco se mantenían a los dos años, aunque se registra un ligero declive en la efectividad de la terapia farmacológica (40), mayor en los que mostraron más beneficios al inicio $(7,34)$.

4.1.2.3. El MTA a los 36 meses

En el 2007 se publicaron los resultados de seguimiento del MTA a 3 años $(28-29,41)$.

Los cuatro grupos de tratamiento mantienen mejoría significativa, pero no hay diferencias entre ellos en ninguna de las áreas evaluadas.

Cualquier resultado a favor de la medicación se desvanece y, al contrario, el uso de medicación durante el periodo de los 24 a los 36 meses se revela como un predictor del empeoramiento sintomático. La interrupción de la medicación a los dos años se asoció a una mejoría posterior (28). Aquellos que habían permanecido con medicación tenían puntuaciones más altas en tendencia antisocial y delincuencia $(28-29,34)$ y a mayor dosis más alta era la tendencia $(27,56)$. En cambio los que procedían del grupo con sólo tratamiento conductual mantuvieron la mejoría y generalizaron las habilidades a otros escenarios.

Como las diferencias entre los grupos desparecieron, los autores investigaron razones alternativas al declive farmacológico. Confrontaron dos hipótesis: a) un sesgo por auto-selección hizo disminuir la puntuación de los medicados, y b) la mejoría se disipó entre diversas trayectorias en los medicados. Su análisis no encontró pruebas a favor de la auto-selección, y aunque observaron ligeros beneficios de la medicación en algún subgrupo, no hallaron la explicación alternativa a la falta de diferencia entre los grupos (65).

Se constató que los medicados habían crecido menos, en talla y peso, respecto a los niños sin TDA/H y a los que no tomaron medicación (41).

\subsubsection{El MTA a los 6 y 8 años}

Los resultados se publicaron en 2009 (30) y se basan en la evaluación de padres, profesores y de los propios participantes (en ese momento alumnos de high school) mostrando que no había nada que indicara que quienes fueron tratados con estimulantes estuvieran mejor que quienes no lo hicieron. A estas alturas del estudio MTA, Molina afirma que no hay pruebas que indiquen el beneficio de mantener la medicación más de dos años (30).

El uso del fármaco se asocia con un resultado peor en hiperactividad e impulsividad, con más signos de trastorno de oposición desafiante y un funcionamiento global peor (30). Myttas afirma que con fármacos mejoraron a los 14-24 
meses, pero después su funcionamiento empeoró y necesitaron más servicios escolares extra a los 36, 48, 72 y 96 meses que quienes no tomaron fármacos (32). Es decir, el uso de estimulantes se asoció a un deterioro en las áreas de impulsividad, hiperactividad, agresividad y psicopatología, medido con la Columbia Impairment Rating Scale, y un peor rendimiento académico y funcionamiento global.

Pappadopulos et al. en un trabajo publicado en el mismo número de la revista argumenta que los niños medicados pudieron engañar y que en realidad no tomaron la medicación un 25\% de ellos en más del $50 \%$ de las veces, a pesar que el $89,8 \%$ de los padres informaban que se cumplía el tratamiento farmacológico (66).

El editorial que acompaña los artículos de Molina y Pappadopulos da por demostrado que los efectos positivos mostrados por el fármaco al inicio del estudio MTA habían desaparecido (31).

\subsubsection{El estudio RAINE}

El "The Western Australian Pregnancy Birth Cohort (Raine) study" (33) comenzó en 1989 como una cohorte de 2868 mujeres embarazadas en su $18^{\text {a }}$ semana de gestación en Perth, Australia Occidental, una región conocida por sus altas ratios de uso de estimulantes (anfetamínicos y metilfenidato) en niños diagnosticados de TDA/H (67). Se recogieron datos de los participantes (tanto madres como hijos) cuando los niños tenían 1, 2, 3, 5, 8, 10, 14 y 17 años, con la particularidad de que quienes los recogían no conocían la utilidad final que se les iba a dar.

A los catorce años 131 de los 1785 adolescentes que continuaban en el estudio habían sido diagnosticados de TDA/H. Se agruparon según 2 criterios:

1.- Uso actual de la medicación. A los 14 años, 21, el 16\%, tomaban medicación y lo habían hecho de forma continua desde el diagnóstico; 40 (30,5\%) tomaban medicación pero no lo habían hecho de forma continua en el pasado; 41 $(31,3 \%)$ la habían tomado pero ya no lo hacían a los 14 años, y $29(22,1 \%)$ nunca la habían tomado.

2. Uso histórico de la medicación. El 16\%, 21 adolescentes, la tomaban en los tres puntos de corte, 8,10 y 14 años, el $32,1 \%$, 42, en dos de ellos, en uno el $29.8 \%$ (39), y se mantuvieron sin medicación el 22,1\%, 29.

En el punto de corte de 5 años ninguno tomaba medicación y no se encontraron diferencias significativas entre los grupos al controlar variables como la severidad de sintomatología TDA/H, otras variables sanitarias, escolares y sociodemográficas. Las halladas más tarde se desarrollaron en el periodo entre los 5 y 14 años, cuando parte de estos niños fueron medicados.

El estudio concluye que:

a) Ninguna mejoría en cualquiera de las variables se asoció al uso de la medicación para el TDA/H.

b) Los que tomaron medicación tenían 10,5 veces más probabilidades de ser identificados por los profesores por un rendimiento escolar menor al correspondiente a su edad. 
ORIGINALES Y REVISIONES

c) Al uso de la medicación se asoció a un incremento significativo de la tensión arterial diastólica de aproximadamente $10,8 \mathrm{mmHg}$. No se trata de un efecto a corto plazo puesto que aquellos que tomaron estimulantes de forma continua en todos los puntos de corte tenían una TA diastólica significativamente superior $(7 \mathrm{mmHg})$ a la de quienes no la tomaron de forma continua en el pasado.

d) Aunque otras diferencias entre los grupos no se hallaron estadísticamente significativas, los síntomas TDA/H y el funcionamiento global fueron peores en los que tomaron medicación (33).

El estudio RAINE 2011 está limitado por el pequeño número de participantes - mayor no obstante al de muchos ECAs a corto plazo- en el que no pudo tenerse en cuenta el subtipo TDA/H, ni el tipo de estimulante utilizado. Según Whitely se trata de resultados que se conocieron en Febrero 2010 y que tardaron casi un año en publicarse; el estudio continúa pero el comité ministerial que analizó los datos y los publicó se disolvió más tarde y no está claro qué sucederá con los datos recogidos en los puntos de corte de 17 y 20 años (68).

\subsection{Sobre la atomoxetina}

La atomoxetina es un inhibidor selectivo de de la recaptación de la noradrenalina (RNI) que, indirectamente, puede aumentar la dopamina en el córtex prefrontal (59). Fue ensayado inicialmente como antidepresivo, sin éxito, y aprobado en 2003 en USA para tratar el TDA/H $(67,69)$. Suele considerarse como un fármaco no estimulante, no sujeto por tanto a las restricciones regulatorias de estos últimos, aunque la OMS (60) lo cataloga como simpaticomimético de acción central, en el mismo grupo que las anfetaminas, el metilfenidato, el modafinilo y otros, y así se recoge en algunos manuales y guías prácticas de psicofarmacología (70-71). En cualquier caso, su perfil de efectos adversos en apetito, frecuencia cardíaca (FC) y tensión arterial (TA) es muy similar al de los estimulantes (3).

\subsubsection{La atomoxetina a corto plazo}

Se comercializó en USA tras la publicación de 11 informes sobre 8 estudios fase II y fase III -todos financiados por la farmacéutica y dos cofinanciados por el NIMH- de entre 6 y 12 semanas de duración (69).

Velásquez Tirado y Peña avalan su eficacia con 5 estudios, el más largo de 9 semanas (72).

Los estudios comparados con placebo a corto o muy corto plazo indican, en general, que el fármaco es más efectivo (3-4,25-26) aunque algunos de estos estudios son metodológicamente criticables (67).

Sus efectos sobre los síntomas nucleares del TDA/H se consideran ligeros o moderados (3). Newcorn et al. en un estudio publicado sobre 618 pacientes y prolongado 9 semanas encontraron que el $40 \%$ de ellos no obtenía beneficio alguno, un $13 \%$ tendría una respuesta pequeña, y el $47 \%$ se beneficiaron del fármaco (73). 


\subsubsection{La atomoxetina a largo plazo}

La GPC española recoge dos estudios para defender el tratamiento a largo plazo con atomoxetina. Ambos comparten investigadores. Uno se centra en niños y el otro en adolescentes, y no sobrepasan los dos años.

Kratochvil et al. evaluaron a 272 niños tratados con atomoxetina, de entre 6 y 7 años, siendo 97 los que se mantuvieron hasta dos años de tratamiento, es decir, el $35,7 \%$. El $25,7 \%$ dejó el tratamiento por falta de eficacia y el $4 \%$ por efectos adversos. Se observó un efecto significativo de retraso en el crecimiento en las primeras fases del tratamiento, que se atenuó posteriormente. Se observó un impacto significativo en la tensión arterial, la frecuencia cardiaca y en la disminución del intervalo $\mathrm{PR}$, sin otros efectos en el sistema cardiovascular. Los investigadores concluyeron que la atomoxetina es útil a largo plazo y, en general, bien tolerada (47).

Wilens et al. se centran en adolescentes. Incluyen 601 sujetos de los que 219 cumplieron el tratamiento de dos años, es decir, el 36,4\%. 99 (16,5\%) lo abandonaron por falta de eficacia y $31(5,2 \%)$ por efectos adversos. Los efectos indeseados fueron menores que en niños. Concluye que los efectos beneficiosos se mantenían (48).

Sólo se ha encontrado un estudio de más de dos años de duración. Se trata de un estudio fase III y IV, realizado por la propia farmacéutica que sigue a los pacientes durante cinco años. Son 1553 pacientes iniciales con una media de edad de 11,6 años. Completaron el estudio, en la fase IV, 67 pacientes. Son 103 pacientes los que completaron toda la fase III. Abandonaron el tratamiento por voluntad propia 432 pacientes, se perdieron en el seguimiento 232, lo dejaron 204 por ineficacia, por efectos adversos 86, y por mejoría satisfactoria 80 pacientes (52).

Se trata de estudios que no comparan resultados con sujetos con TDA/H sin fármacos. Un gran porcentaje de sujetos abandona el tratamiento, lo que apunta a que, posiblemente, no percibían grandes ventajas y este es un dato que puede cuestionar los resultados obtenidos en el estudio.

\subsection{Metilfenidato versus atomoxetina}

King et al. citan únicamente dos estudios, uno que no encuentra diferencias entre ambos a altas dosis, y otro que considera superior el metilfenidato con ambos fármacos a dosis medias (25).

La GPC NICE considera que, al compararse con el placebo, el efecto del metilfenidato es mayor que el de la atomoxetina. Cita dos estudios que comparan ambos productos. El primero encuentra a la atomoxetina "no inferior". El segundo muestra que el metilfenidato de liberación retardada es superior (3).

La GPC SIGN cita un metaanálisis que encuentra inferior a la atomoxetina y 6 estudios comparativos, de los cuales dos consideran su efectividad similar (metilfenidato de liberación inmediata), dos encuentran el metilfenidato superior (liberación prolongada), uno encuentra la atomoxetina "no inferior" (metilfenidato de 
ORIGINALES Y REVISIONES

liberación inmediata) y otro, el metilfenidato superior (metilfenidato de liberación prolongada) (4).

La GPC española cita a la NICE y recoge un metaanálisis y una revisión sistemática para sugerir una eficacia mayor del metilfenidato sobre la atomoxetina (5).

El informe Drug ERPO cita dos estudios en niños de edad escolar (uno de ellos ya citado por la NICE) que consideran la atomoxetina no inferior o equivalente al metilfenidato de liberación inmediata. Un ensayo clínico de 6 semanas de no inferioridad encuentra el metilfenidato de liberación prolongada superior a la atomoxetina (26).

Aunque los estudios son escasos, parece extendida la opinión de que la atomoxetina es menos efectiva que el metilfenidato.

5. Mecanismo de acción presumido, neuroadaptación, síndrome de discontinuidad, naturaleza de los cambios atencionales producidos por los fármacos y experiencia subjetiva de su toma

\subsection{Mecanismo de acción, neuroadaptación y síndrome de discontinuidad}

Partiendo de la acción del metilfenidato se ha hipotetizado como causa del TDA/H algún tipo de desequilibrio en la neurotransmisión dopaminérgica y noradrenégica en el sistema nervioso central (2) y en particular en el córtex prefrontal (59) que sería revertido por los fármacos. Si los fármacos funcionan en niños con TDA/H ello "indica que son deficientes en arousal cortical y autónomo" (74). Parece tratarse de una falacia "post-hoc" y, además, olvida que no hay ninguna paradoja en el efecto sobre la atención asociado a la toma de estimulantes puesto que todos ellos, en dosis moderadas, aumentan la atención, incluyendo la cocaína, la cafeína y otros (54) y que no se ha hallado hasta el presente prueba alguna de desequilibrio químico en el TDA/H (27).

La neuroadaptación es el proceso por el que el sistema nervioso central reacciona a la toma de psicofármacos contrarrestándolos. En el proceso de neuroadaptación al fármaco se dan cambios en las vías de señalización intracelular y expresión génica, y el cerebro del paciente funciona de una manera que "tanto cualitativa como cuantitativamente es diferente del estado normal" (75). Implica a neurotransmisores, neurorreceptores, enzimas, producción y almacenaje de los neurotransmisores, su recaptación, hormonas... y en el caso de los estimulantes provoca cambios, como la disminución de los transportadores de dopamina, disminución de receptores de la dopamina, alteraciones en la expresión génica reduciendo la expresión de c-Fos en el cuerpo estriado, y otros cambios morfológicos (75). Dicho de otra manera, es muy probable que los efectos a corto y largo plazo 
del metilfenidato sean muy diferentes, incluso contradictorios, habiendo llegado a hipotetizarse que es posible que, a los meses de tomar estimulantes, haya menos dopamina disponible que antes de iniciar el tratamiento (35). Suponer que la toma de psicofármacos produce efectos similares durante años no tiene base neurobiológica.

Un pequeño estudio en niños con TDA/H mostró que, a los tres meses de exposición, al metilfenidato se habían reducido en un $20 \%$ los receptores dopaminérgicos D2. También disminuyeron los transportadores de dopamina en un 74,7 \% en el estriado. Uno de los niños fue estudiado al mes de interrumpir la medicación que mantuvo durante un año, observándose un aumento de los trasportadores entre el 30-50\% en el estriado izquierdo respecto a su estado previo al tratamiento (76). Puede tratarse de la base neurobiológica del bien conocido síndrome de discontinuidad (54), caracterizado por el empeoramiento notable de la sintomatología tras la interrupción brusca de la medicación estimulante (77-79). Puede que el organismo necesite tiempo, tal vez mucho, para neuroadaptarse de nuevo, esta vez a la ausencia de medicación, y el riesgo de obtener conclusiones clínicas precipitadas de la interrupción brusca y durante breve periodo de la medicación estimulante es grande.

5.2. Naturaleza de los cambios atencionales producidos por los estimulantes

Los estimulantes estrechan el campo atencional. En ese sentido, disminuyen la curiosidad, el afán exploratorio, la espontaneidad, la capacidad de asombrarse y preguntar, el atender a numerosos estímulos y dispersarse, redireccionar la atención y el interés, la flexibilidad cognitiva y el cambio emocional $(7,80)$. A dosis bajas los humanos y los animales muestran inhibición en la conducta exploratoria espontánea, reducen su interés sobre el entorno, y disminuyen las interacciones sociales.

Los animales bajo los efectos del metilfenidato y otros estimulantes muestran comportamientos repetitivos, excesivamente centrados, conductas sin sentido, como rascarse, excesiva limpieza, roer o mirar fijamente a pequeños objetos $(54,81)$.

La neuropsicóloga Betina Gardner refiere un beneficio del metilfenidato en la atención sostenida, pero no en la atención selectiva y la dividida, y ello confirma lo hallado por otros investigadores (82).

Por otra parte, la atención es una facultad que se desarrolla con el crecimiento y maduración del niño a medida que la dispersión se desvanece, algo que también parece ocurrir en los animales. Muchos de los niños medicados lo están cuando la maduración de la atención está en ciernes (83).

\subsection{La experiencia subjetiva de la toma de metilfenidato}

Hay pocos estudios sobre la experiencia subjetiva de tomar psicofármacos de cualquier tipo, exceptuando las benzodiazepinas (54-55).

De los estimulantes hay referencias de quienes los toman de forma lúdica. 
ORIGINALES Y REVISIONES

Ningún estudio sistemático y riguroso se ha hecho relacionado con la toma de metilfenidato en pacientes.

La GPC NICE presenta un estudio sobre lo que experimentan los niños diagnosticados de TDA/H bajo tratamiento con estimulantes, centrado en su capacidad de discernir cambios positivos en su conducta, efectos secundarios, valorar molestias/beneficios de la toma de medicación y del estigma asociado. Dice poco acerca de la experiencia subjetiva de la toma de estimulantes salvo que "su valoración positiva no significa que les guste tomar medicación” y que "los niños mayores suspiran porque llegue el momento en que puedan prescindir de las pastillas” La misma guía señala la tendencia, bien conocida por los clínicos, de interrumpir la medicación al llegar a la adolescencia (3).

La GPC española cita entre los efectos secundarios del metilfenidato la "falta de espontaneidad" (5).

A los niños no parece gustarles el fármaco y expresan que se sienten infelices y que querrían estar como antes de tomarlo (84).

Un estudio que recogió las opiniones de los niños vio que, aunque rara vez se quejaban a sus médicos, tenían un rechazo generalizado a la toma del estimulante, y describían su experiencia con expresiones como "Me atonta", "Me hace triste", "Nada me hace reír", "Se apodera de mí" o "No me hace sentirme como soy" (85).

Teniendo cuenta los efectos atencionales descritos más arriba parece que estamos frente a un declive de la actitud y actividad propia de la infancia, sobre la que los niños construyen su personalidad y su estar en el mundo. Es fácil de entender que la disminución de la curiosidad, la espontaneidad o el disfrute no sea apreciada por los niños.

Un dato que apoya la idea de que los menores no aprecian positivamente la toma de estos fármacos es la relevante tasa de incumplimiento de la prescripción, que puede oscilar entre el $20 \%$ y el $65 \%(86-87)$.

\section{Riesgos y efectos adversos del metilfenidato y de la atomoxetina}

\subsection{Los riesgos del metilfenidato}

Hay varios efectos a considerar en la toma mantenida de metilfenidato en diferentes sistemas:

1. Efectos cardiovasculares. El metilfenidato puede aumentar la tensión arterial y la frecuencia cardiaca. El estudio RAINE lo muestra de forma clara como efecto a largo plazo. Tras el fallecimiento de algunos niños por pro- 
blemas cardíacos la FDA americana llegó a proponer en 2006 una alerta -Black-Box Warning- finalmente rechazada por considerar que padecían un defecto coronario congénito (24). Pero algunos de ellos no tenían ese defecto y el riesgo cardiovascular asociado a la toma de estimulantes es bien conocido (55). Un estudio reciente, financiado por el Instituto de Salud Mental de USA (NIHM), indica que bajo el fármaco se multiplica por 6 ó 7 la posibilidad de muerte súbita (88). La idea, a veces manifestada, de que no es un problema el incremento de la tensión arterial (89) no resulta aceptable.

2. Efectos endocrinos. No hay referencias frecuentes al impacto hormonal del metilfenidato y no existen estudios de su impacto sobre la pubertad en humanos. Se sabe que puede interferir en muchos de los sistemas hormonales. La propia dopamina y sus fluctuaciones influyen en el sistema hormonal de muchas formas. De forma directa o indirecta, el metilfenidato puede estar implicado en la homeostasis endocrina y se le supone un impacto sobre la tiroxina, bastante reconocido, sobre la prolactina, la hormona del crecimiento (81), las hormonas sexuales, la vasopresina y la insulina. Un estudio reciente sobre macacos pre-púberes encuentra pruebas sólidas de que el metilfenidato retrasa de forma patente el desarrollo de la pubertad (36). Aún con todo, los estudios hoy muestran datos contradictorios (20, 34-35, 90).

3. Efectos sobre el crecimiento. El debate acerca del crecimiento de los niños medicados con metilfenidato está desapareciendo, reconociéndose la menor talla y peso de los tratados a largo plazo $(20,35,41,91)$. Es muy posible que no se trate únicamente de un problema de talla y peso asociado al poco apetito que impide la ingesta de los nutrientes necesarios, como se defiende a veces $(3,5)$, sino un fenómeno de retraso en el programa de desarrollo madurativo del niño que puede incluir a órganos como el cerebro, tal vez relacionado con el impacto sobre la tiroxina y la hormona del crecimiento $(20,35,92)$. También la formación del cartílago es retardada, según estudios en vitro $(20,93)$. Se ha afirmado que después de dejar el fármaco hay un rebrote en el crecimiento, efecto estirón, y, aunque fuera cierto, no se trata de un crecimiento normal, ya que el programa de crecimiento y maduración avanza a etapas.

4. Efectos sobre el sueño. Es uno de los efectos secundarios más frecuentes y conocidos aunque suele considerarse un inconveniente menor y manejable (3-5) cambiando pautas posológicas o formas de liberación del metilfenidato. No obstante, su alcance, significado y relación con el TDA/H son objeto de discusión.

Hay quienes afirman que el problema en el sueño se debe al propio TDA/H 
(94) y que el tratamiento no lo afecta negativamente (95-96). Incluso se ha pensado que algunos problemas del sueño en preescolares son un buen predictor de lo que posteriormente será visto como TDA/H, mejor aún que el nivel de hiperactividad (94,97-98,). Sea como fuere, la relación entre medicación estimulante y alteración del sueño se considera sólida (98). Si los niños con TDA/H muestran problemas con el sueño, tales como pesadillas, sobresaltos, dificultad para conciliar el sueño, mayor latencia para el inicio del sueño REM, disminución del sueño REM, el estudio de O'Brien (p 239-240), aún sin pretenderlo, muestra que el sueño REM disminuyó más en los grupos que tenían más niños medicados $(20,35,94)$. Cuando se trata el TDA/H con estimulantes, entre el 30 y $50 \%$ de los niños muestra problemas significativos de sueño al inicio del tratamiento y el $20 \%$ seguía con ellos al año de permanecer en tratamiento (99). En modelos animales se ha observado que el metilfenidato puede modificar el patrón del ritmo circadiano según un modelo dosis-dependiente (100). Los autores de este estudio dan por demostrado que los ensayos que comparan placebo y estimulantes muestran que estos empeoran el sueño, incrementan su latencia, lo hacen menos eficiente y disminuyen su duración, aumentando el movimiento, alterando la arquitectura del sueño y los ritmos circadianos. Recuerdan que algunos signos del TDA/H pueden empeorar con un sueño de mala calidad y piensan que el metilfenidato podría agravarlos (100). Mantener el patrón del sueño se considera importante para el desarrollo hormonal, el neurodesarrollo, el aprendizaje y la memoria, y el crecimiento (20) y se sabe que los problemas de sueño se relacionan con déficit en las funciones superiores, como la flexibilidad cognitiva, el razonamiento y el pensamiento abstracto, y con otros procesos como el metabolismo (100).

De cualquier modo, el dormir es un indicador sensible a las dificultades y el estrés relacional, al igual que la atención. El mismo estrés ambiental puede impactar en la maduración en general y en la atención en particular. La capacidad de dormir se asocia también con la autorregulación, otro factor muy sensible al apego, a la calidad del espacio interactivo y a la estimulación del entorno, también al más puramente perceptivo como los niveles de ruido. Que este tipo de indicadores somáticos, como alteraciones en el sueño, concurran en los niños no asegura de que se deban a una anomalía biológica subyacente, como lo muestran la neuropsicólogo Ruth Schmidt Neven y sus colaboradores cuando, de forma minuciosa, exponen el desarrollo atencional en los niños (83).

5. Sobre el sistema nervioso. A través de estudios con animales se cree que pueden darse cambios neurobiológicos permanentes asociados a la exposi- 
ción temprana a los estimulantes $(20,35,58,101)$. Urban et al. indican que el córtex prefrontal es muy sensible al metilfenidato, y que el tratamiento con este fármaco puede inducir cambios duraderos, incluso permanentes, en la capacidad excitatoria o hipersensibilización de las neuronas prefrontales, tal y como ocurre en los animales sujetos de su estudio $(37,101-102)$. Estudios con modelos animales muestran que retarda el crecimiento de las dendritas, disminuyendo la conectividad neuronal, la mielinización del sistema nervioso, y que daña la sustancia gris $(20,35)$. Un metaanálisis reciente muestra que la mayor densidad del transportador de la dopamina en estriado, un $14 \%$ en el TDA/H, se asocia a la exposición previa al estimulante, y que los TDA/H que no lo han tomado nunca tienen una densidad menor, siendo también menor en los sujetos sanos (103). Los autores justifican su estudio al existir referencias que afirman mayor y menor número de transportadores de la dopamina en el TDA/H. Este estudio ha provocado un interesante intercambio de críticas (104-105).

La evaluación realizada por la psiquiatra y neurotoxicóloga Grace Jackson de datos obtenidos en diferentes estudios longitudinales de índole neurobiológica con neuroimágenes en TDA/H de Castellanos (106), Mackie (107) y Shaw (108-110), sugiere que hay daño cerebral por exposición a estimulantes de forma mantenida (ver Tabla 1) (35).

6. Sobre la escolarización. Los resultados a largo plazo indican de forma clara que tendrán más dificultades escolares y necesitarán más apoyos los medicados que los diagnosticados de TDA/H que no hayan sido medicados, como se ha indicado antes (30,32-33).

7. La evolución del comportamiento. Hay cierta polémica sobre esta cuestión, pero existen estudios, incluyendo el MTA, que muestran que los niños medicados a largo plazo presentan más riesgo de uso de drogas (43-44,30, 111) y puntúan más alto en las escalas de comportamiento desafiante, delincuencia y tienen peor funcionamiento global (30). Aún es más claro que no existen pruebas de que los medicados tengan ventaja en estos aspectos (26). Todo esto debería requerir estudios adecuados, rigurosos y metodológicamente exigentes sobre esta cuestión (54).

8. Otros efectos. Un 8-9\% de los niños que toman metilfenidato presentan tics y otros movimientos anormales (112-113) un efecto bien conocido a dosisdependiente de los estimulantes $(54,81)$, aunque hay estudios que matizan la asociación (114-115). Hay estudios que muestran que el 7\% tuvo crisis psicóticas (116) y un número similar pueden mostrarse más irritables y menos capaces de comportarse bien (34). En USA, otros estudios indican que el $11 \%$ de los niños tratados será diagnosticados posteriormente de trastorno bipolar (117), lo que plantea el debate de si se trata de comorbilidad, error diagnóstico o iatrogenia. 
Tabla 1:

Estudios longitudinales con neuroimagen sobre el TDA/H.

Revisados e interpretados por Grace E. Jackson (35)

\section{Castellanos et al. 2002 (106)}

152 niños con TDA/H (49 de ellos sin tratamiento farmacológico previo) versus 139 controles.

Edad entre 5 a 16 años en niñas, y de 5 a 18 1/2 en varones.

Estudio con diseño muy defectuoso.

La exposición previa a estimulantes se asoció con el núcleo caudado más pequeño, crecimiento menor del cerebelo y capacidad verbal inferior. El tratamiento farmacológico pasado, reciente o continuado se asoció a un enlentecimiento persistente del desarrollo del cerebro. Tras la pubertad se manifestó atrofia cerebelosa.

Mackie et al. 2007 (107)

Estudio con neuroimagen RMN de 36 niños con TDA/H y 36 controles.

Edad de 10,5 años al inicio y 16,5 años al final.

3 o más neuroimágenes cerebrales por sujeto, a intervalos de tres años.

El 94\% estaba tomando medicación en la ultima neuroimagen.

Los niños expuestos a estimulantes tuvieron peores puntuaciones en el área social, académica y en conducta.

Los niños bajo estimulantes presentaban déficits persistentes en el volumen del vermis y atrofia postpuberal del cerebelo.

\section{Shaw et al. 2007 (108)}

Estudio longitudinal con neuroimagen RMN (timing de maduración cerebral)

223 sujetos con TDA/H y 223 controles.

Edad entre 5 a 20 años.

El 25\% fue sometido a repetidas neuroimágenes en un intervalo de tres años.

El $66 \%$ de los TDA/H había estado expuesto a estimulantes anteriormente.

La maduración del córtex de los TDA/H se retrasó alrededor de unos tres años.

(Maduración = edad de aparición del grosor máximo).

\section{Shaw et al. $2006(109)$}

Estudio longitudinal y transversal con neuroimagen RMN (grosor máximo del córtex).

163 sujetos con TDA/H y 166 controles.

Edad de 10 a 10,5 años $>16,5$ a 18,5 años.

El $60 \%$ de los sujetos tuvo más de una neuroimagen.

El $66 \%$ de los menores con TDA/H había usado estimulantes.

Los sujetos con uso pasado, reciente o continuo de estimulantes nunca alcanzaron los niveles de grosor cortical de los sujetos del grupo control.

\section{Shaw et al. $2009(110)$}

Estudio longitudinal con neuroimagen RMN.

43 sujetos con TDA/H, de entre 12 a 17 años.

Las neuroimágenes se obtuvieron en un intervalo de de 4 años.

19 niños interrumpieron el uso de estimulantes, 24 continuaron con ellos.

Los niños que interrumpieron el tratamiento tuvieron mayor éxito:

- $\quad$ en alcanzar la remisión completa del TDA/H.

- en evitar otras terapias farmacológicas.

- $\quad$ en el proceso acelerado de adelgazamiento cortical postpuberal.

\subsection{Los riesgos de la atomoxetina}

El conocimiento de sus efectos indeseados es más limitado dada su más reciente comercialización (3). Dolor abdominal, náuseas, vómitos, disminución del apetito, pérdida de peso, daño hepático, incremento del intervalo PR son efectos comunes de un fármaco con el que es necesario monitorizar crecimiento y desarrollo y que puede producir o empeorar actitudes suicidas, hostilidad y labilidad emocional (118-119). 
También conlleva problemas con el sueño, aunque pueden ser menores que con el metilfenidato (98).

Sus efectos cardiovasculares conllevan aumento de la frecuencia cardiaca y es muy común que incremente la tensión arterial, pudiendo llegar a ser permanente, incluso en niños, según la Agencia Española del Medicamento (120).

La posible agresividad del menor bajo su toma fue establecida en un estudio realizado por la empresa farmacéutica en menos de un $2 \%$ (121). También se ha referido mortalidad por su uso, cuestión hoy en estudio por la FDA. La MHRA (Medicines and Healthcare products Regulatory Agency) en Inglaterra llama la atención sobre efectos psiquiátricos adversos, tipo manía o psicosis (122).

Su potencial de abuso no parece haber sido estudiado exhaustivamente. Abad et al. citan un estudio en adultos comparado con placebo para descartarlo (118). Modelos animales apuntan a su no discriminación con dosis bajas de cocaína (123). Respecto a la experiencia subjetiva al tomarlo, Heil et al. sugieren que la ingesta de atomoxetina se diferencia poco de la del placebo e incluso en dosis altas no tendría efectos placenteros, por lo que sería menos proclive a transformarse en una sustancia de abuso (124). Se ha hipotetizado que ello podría deberse a que la atomoxetina, contrariamente al metilfenidato, no tiene impacto sobre los niveles de neurotransmisores en el estriado y en el núcleo accumbens (125).

6.3. Riesgos comparados de metilfenidato con atomoxetina

Los autores que comparan estos dos fármacos en referencia a sus efectos indeseados son contradictorios. Algunos estudios los consideran similares, como el metaanálisis de Hanwella et al (126).

Wang et al. presentan un estudio multinacional con 339 niños y muestra que los efectos adversos con la atomoxetina son más frecuentes que con el metilfenidato (ver Tabla 2), de intensidad entre media y moderada, e indica que tienden a disminuir con el tiempo. No encontró diferencias en referencia al intervalo QT, tensión arterial y frecuencia cardiaca entre ambos fármacos (127).

Tabla 2:

Efectos adversos comparados entre atomoxetina y metilfenidato (127)

\begin{tabular}{|l|c|c|}
\hline & Atomoxetina & Metilfenidato \\
\hline Pérdida de peso & $1,2 \mathrm{Kg}$. & $0,4 \mathrm{Kg}$. \\
\hline Anorexia & $37 \%$ & $25 \%$ \\
\hline Náuseas & $20 \%$ & $10 \%$ \\
\hline Somnolencia & $26 \%$ & $4 \%$ \\
\hline Vértigos & $15 \%$ & $7 \%$ \\
\hline Vómitos & $12 \%$ & $6 \%$ \\
\hline
\end{tabular}


ORIGINALES Y REVISIONES

La valoración realizada en Columbia University alerta de los efectos indeseados de la atomoxetina y recomienda considerarla sólo para los pocos casos en los que otros fármacos no funcionen, debido a sus riesgos (119).

\section{Discusión y conclusiones. El caso de las Guías de Práctica Clínica}

"Los niños merecen algo más que una escala y medicación” Lidia FURMAN (24)

\subsection{El caso de las GPC}

En su acerada crítica a la GPC NICE, Double y Timimi recuerdan la "Jerarquía de Evidencias" que deberían respetar las GPC a la hora de realizar sus recomendaciones, 1) mortalidad, 2) resultados adversos graves, como hospitalización, adicciones, criminalidad, etc.; 3) resultados a largo plazo en términos de funcionalidad, como años de escolaridad completados, resultados académicos, etc.; 4) efectos adversos a corto plazo; y 5) efectos beneficiosos a corto plazo en la esfera conductual, cognitiva etc. El balance ha de ser satisfactorio para que el fármaco pueda ser recomendado (53).

Esta jerarquía no se respeta en el TDA/H. Las GPC construyen sus recomendaciones sobre ensayos clínicos ECA a corto plazo extremadamente criticables en lo que se refiere a su metodología, recogida de datos, detección de efectos adversos y evaluación de resultados en términos de funcionalidad $(25,26)$. De los metaanálisis y revisiones sistemáticas consultadas, sólo en Drug ERPOy King et al. se consideran sistemáticamente aspectos de funcionalidad $(25,26)$. Respecto a lo que pueda suponer desde una perspectiva de salud pública a largo plazo el uso de estimulantes en TDA/H en términos de funcionalidad/discapacidad social, es necesario acudir a otras fuentes (57).

Todo sucede, en el TDA/H y en otras áreas, como si la medicina basada en la evidencia se hubiera caricaturizado al basarse en estudios ECA a corto plazo, de pobre calidad, centrados en la supresión de síntomas más que en la recuperación y la mejoría funcional, proponiendo estándares de cuidados escasamente relevantes para pacientes con problemas de disfuncionalidad importantes y tratados normalmente a largo plazo (34). Es un fenómeno particularmente extendido en psiquiatría a otras categorías diagnósticas y fármacos (128).

En el TDA/H se hipotetiza que el control de la sintomatología nuclear está altamente correlacionado con la mejoría funcional (2). Nada más lejos de la realidad, al menos en lo que se refiere a los resultados de los estudios a largo plazo. 
El estudio a más largo plazo que recoge la guía española y sus modelos $(3,4)$ es el MTA a los 3 años, publicado en 2007. No obstante, la guía NICE (p. 334) reconoce que las recomendaciones sobre el uso de metilfenidato a largo plazo se basan únicamente en los estudios a 14 y 24 meses (3) y critica el estudio MTA a los 3 años que, recordemos, afirmaba que "en el seguimiento a los 3 años no se obtienen diferencias significativas entre los grupos con medicación o sin ella", interpretando los resultados de forma más optimista que los propios autores del estudio. La GPC española recoge estas críticas afirmando que "los resultados deben interpretarse con cautela debido a que el seguimiento tras la intervención es naturalístico, sin controlar la intervención, y a la ausencia de un grupo control sin tratamiento". Se trata de aspectos que en gran parte caracterizan el estudio MTA desde su comienzo, ausencia de grupo control, estudio no cegado... que no impide a la GPC española considerar las pruebas del MTA a 14 y 24 meses como de máxima calidad científica (5).

Sin embargo, como señalan Double y Timimi, los resultados del MTA a 3 años no han podido ser explicados mediante hipótesis de convergencia de tratamiento en los grupos o autoselección de los casos más graves para la medicación y, sobre todo, son congruentes con los obtenidos a los 14 y 24 meses (53). Los propios investigadores concluyeron que no encontraron base para hipótesis de convergencia o de autoselección (65).

A los 14 meses, las ventajas atribuidas a la medicación lo eran, fundamentalmente, en síntomas nucleares y, escasamente, en aspectos de funcionalidad. Los peores resultados los obtenía el grupo en la comunidad en el que la mayoría de pacientes estaba bajo tratamiento medicamentoso bajo un formato "no intensivo", muy diferente al grupo medicado del MTA, y similar, probablemente en frecuencia de contactos y atención clínica, al posterior seguimiento naturalístico una vez terminado el ensayo clínico. De hecho, las páginas NIMH en las que se recoge los resultados a 3, 6 y 8 años del MTA lo describen como "Tratamiento Intensivo a Corto Plazo" (129-130).

A los 24 meses, los resultados de los pacientes medicados empeoran ligeramente respecto a las evaluaciones obtenidas a los 14 .

A los 3 años no se observan diferencias significativas entre los grupos y el uso de medicación se considera como un predictor de empeoramiento sintomático y de conducta disocial y delincuencia, y se constató que los medicados habían crecido menos, en talla y peso. Todo ello según los investigadores del MTA.

A los 6 y 8 años, en estudios no recogidos por las guías inglesa y española, el uso del fármaco se asocia con un resultado peor en hiperactividad e impulsividad, con más signos de trastorno de oposición desafiante y un peor funcionamiento global y mayor necesidad de servicios escolares. En cualquier caso, como reconoce el NIMH, no hay ningún dato a favor del uso del metilfenidato a largo plazo (130). 
ORIGINALES Y REVISIONES

El estudio RAINE ratifica la falta de mejoría atribuible al fármaco, los peores resultados en términos de funcionalidad y rendimiento escolar y el aumento de la TA a largo plazo entre los medicados (33).

En resumen, el apartado 3) de la jerarquía de evidencias queda malparado en lo que se refiere al metilfenidato.

Respecto a los efectos adversos a medio y largo plazo, la literatura recoge la posibilidad de efectos adversos graves, puntos 1) y 2) de la jerarquía de evidencias (2), y se muestra mucho más discreta respecto a la intensidad y relevancia de otros efectos adversos, como las alteraciones del sueño, el retraso en el crecimiento y los cambios cardiovasculares. En realidad, la crítica a los ECAs realizada respecto a aspectos de eficacia y efectividad es extensible a la detección y valoración de efectos adversos (25) y los estudios sistemáticos sobre posibles cambios hormonales, retraso de la pubertad, de maduración o de experiencia subjetiva de la toma de metilfenidato brillan por su ausencia.

En líneas generales, y considerando tanto la valoración de efectos beneficiosos como adversos, la afirmación sobre el metilfenidato, tan al uso entre nosotros, de que "posiblemente no exista un fármaco más y mejor estudiado de los que se usan en edad pediátrica que el metilfenidato" (89) no tiene base alguna.

Se presenta en la Tabla 3 un resumen de las recomendaciones de las GPC inglesa, escocesa, española y australiana.

Haciendo una comparativa de GPC, la australiana, que recoge los MTA a 8 años, el estudio RAINE, al igual que el Drug ERPO, es la que menos indicaciones facilita a la hora de optar por un tratamiento u otro. La inglesa, modelo de la española, se basa en el MTA a la hora de proponer el metilfenidato como tratamiento de elección en los casos graves -cita un estudio complementario del MTA a 14 meses realizado años después (131)- y fundamenta su apoyo a la terapia combinada en los resultados del MTA a 14 meses. Facilita un punto de corte para los casos severos (el diagnóstico hipercinético del ICD10) y aconseja tratamiento psicológico con carácter inicial para el resto de TDA/H para cuyo diagnóstico considera necesaria una disfuncionalidad de moderada a grave (3). La guía española es, de lejos, la más optimista de todas a la hora de indicar el tratamiento con fármacos como la primera elección, y prácticamente para todos los casos, puesto que su definición de TDA/H leve es la de aquel en el que apenas existe disfuncionalidad. Considera en la práctica el metilfenidato y la atomoxetina como fármacos de primera elección, a pesar de hacerse eco de la opinión a favor del primero de la inglesa y escocesa y recoger estudios ECA en el mismo sentido (5). 
Tabla 3:

Guías de Práctica Clínica (GPCs), recomendaciones y otros aspectos.

Se muestran, en primer lugar, la NICE y la SIGN, principales fuentes de la guía española, luego ésta y la NHMRC, que se presenta como actualización de las anteriores.

\section{GPC NICE, 2009, Inglaterra (3)}

-Criticada por no mostrar claramente posibles conflictos de interés.

-EL TDA/H es una mala adaptación, disfuncionalidad, derivada de la impulsividad, atención y/o hiperactividad. Es necesaria una disfuncionalidad moderada para su diagnóstico. Se trata de rasgos donde se da un continuum en la población general. No implica necesariamente un trastorno neurobiológico.

-Presenta Metanálisis y Revisiones Bibliográficas Sistematizadas de elaboración propia.

-Se basa, para el uso del metilfenidato, en el MTA a los 14 y 24 meses y 3 años, que cita continuamente y al que dedica 2 largos capítulos, aunque lo critica metodológicamente.

- En el TDA/H de severidad leve (disfuncionalidad moderada) y moderada (disfuncionalidad grave) recomienda como primera opción la intervención psicológica.

- En el TDA/H de severidad grave (que identifica con el tipo hiperactivo según criterios ICD-10) se recomienda psicofarmacología como primera opción junto con entrenamiento parental (basa la recomendación en el estudio MTA a los 14 meses). Considera que en estos casos los resultados con medicación son más rápidos que la intervención psicológica sola y/o los padres no pueden aplicar instrucciones conductuales salvo rápida mejoría obtenida con medicamentos.

- Considera bien establecida la efectividad en el TDA/H de la terapia cognitivo-conductual, entrenamiento en habilidades sociales, entrenamiento parental y uso de manuales de autoayuda. Son eficaces en edad preescolar y niños mayores, en intervención en niños, en padres, en ambos (terapia familiar), en niños con o sin medicación, en formato grupal y en individual. Son procedimientos eficientes, de coste-efectividad favorable, sobre todo en formato grupal. Los procedimientos de psicoterapia se muestran efectivos en áreas más amplias que los síntomas nucleares, como por ejemplo asertividad y resolución de problemas.

- Recomienda tratamiento psicológico y entrenamiento parental (terapia combinada) siempre que se utilicen fármacos, bien sea de primera intención o tras fracaso de tratamiento psicológico solo. Hace hincapié en el interés de la terapia combinada en los casos graves, a pesar de los resultados del MTA.

- No recomienda fármacos a niños menores de 6 años.

- Considera fármacos efectivos el metilfenidato y la atomoxetina.

- Los efectos de la medicación se centran en los síntomas nucleares del TDA/H. Sus efectos son limitados en áreas como la dinámica familiar, la autoestima, las relaciones entre pares y otras. Considera no demostrada la eficacia de los fármacos en resultados académicos y aprendizaje.

- Los fármacos no son efectivos en todos los casos y hay un porcentaje importante que interrumpe el tratamiento por efectos secundarios (15\% a los cuatro meses).

- El fármaco de primera elección es el metilfenidato por resultados superiores a la atomoxetina y menor secundariedad. Recomienda atomoxetina si el metilfenidato no es bien tolerado.

- Cree fundada la idea de que el beneficio de los fármacos se mantiene a largo plazo. En el caso del metilfenidato se basa en el seguimiento MTA a 24 meses, considerando los resultados del estudio a 3 años de manera más favorables al metilfenidato que lo que dicen los propios autores del estudio.

- Considera dudoso el impacto del metilfenidato y de la atomoxetina en la talla y peso por diferencias no significativas en el MTA, datos contradictorios en otros estudios y efecto "estirón". No obstante, recomienda monitorización en talla y peso. Si hay afectación significativa, recomienda vacaciones terapéuticas durante las vacaciones escolares. Recomienda adaptación de la dieta. No contempla la posibilidad de retraso madurativo. No contempla interrupción de la medicación por afectación de la talla y el peso.

- No recoge el posible impacto sobre el sistema hormonal.

- El metilfenidato y la atomoxetina requieren monitorización en TA y FC. Facilita criterios para reducir dosis y derivar a médico de familia si hay alteraciones significativas.

- Si el fármaco tiene una respuesta efectiva debe mantenerse indefinidamente revisando resultados anualmente y considerando qué sucede si se olvidan dosis o hay periodos breves de no tratamiento. No recomienda en general vacaciones terapéuticas.

- Si no hay respuesta favorable a psicoterapia, intervención parental, metilfenidato y atomoxetina, considera, además de revisar aspectos diagnósticos, adherencia al tratamiento, circunstancias familiares y escolares, etc. el uso de dosis de medicación mayores que las que figuran en ficha técnica.

- Entre las recomendaciones de investigación futura incluyen un ECA con interrupción de tratamiento a los 18 meses para obtener evidencias de si el tratamiento a largo plazo es eficaz y seguro.

A lo largo del texto se recogen preocupaciones éticas, necesidad de tener en cuenta la opinión del menor y su familia, explicarles las diferentes opciones y obtener un consentimiento informado de calidad (Información veraz, suficiente y comprensible, junto al respeto del terapeuta a la capacidad de decisión del paciente y/o sus allegados). 


\section{GPC SIGN, 2009, Escocia (4)}

-Realizada por una red de expertos, sin declaración de conflicto de interés.

-Actualización de la Guía de 2001 y otras posteriores, como la de 2005 citada por la GPC Española.

-Dedica un capítulo monográfico al MTA. Recoge el follow-up a 6 y 8 años del MTA.

-Estructura de niveles de evidencia y grados de recomendación idéntica a la GPC Española.

-Etiología multifactorial, aunque subraya importancia de una base biológica y genética.

-Resalta aspecto dimensional de los signos TDA/H, con el subtipo hiperactivo (ICD-10) como más grave. Creen que habría niños y jóvenes no diagnosticables según criterios DSM-IV y CIE-10 que pueden experimentar dificultades significativas. -Realiza recomendaciones off-label y las justifica, remitiendo no obstante a la responsabilidad del prescriptor.

- Para niños de preescolar recomienda entrenamiento conductual de padres (alusión a posibles problemas de desarrollo cerebral por uso de estimulantes en niños de edad preescolar).

- Recomienda fármacos como primera opción para la forma más grave del trastorno, tipo hiperactivo ICD-10. Se basa en el MTA a 14 meses.

- Si los síntomas del TDA/H no son severos, recomienda considerar terapia conductual como primera opción y evaluar resultados utilizando medicación si no son buenos.

- Para todas las edades y subtipos considera el manejo de contingencias escolares e intervenciones académicas más eficaces que las estrategias cognitivo-conductuales.

- En caso de comorbilidad con trastorno de oposición desafiante, agresividad, y ansiedad generalizada recomienda terapia combinada (fármacos más tratamiento conductual). Se basa en el MTA a 14, 24 y 36 meses.

- Considera eficaces el metilfenidato y la atomoxetina. Considera mayor la eficacia y rapidez de la acción del metilfenidato respecto a la atomoxetina, que sería una opción posterior, en caso de fracaso del primero o por reacciones adversas.

- Considera eficaces a 2 años el metilfenidato (MTA a 14 y 24 meses; los califica de estudios de alta calidad) y la atomoxetina a 2 años.

- A más largo plazo, considera "equipotenciales" las intervenciones farmacológicas y las psicológicas (MTA a 8 años).

- Describe efectos adversos de la medicación sobre FC y TA, y recoge posibilidad de que puedan producir retraso en el crecimiento (MTA a 36 meses) aunque no habría datos sobre la altura final en el adulto.

- Recomienda monitorización de FC, TA, talla y peso, aunque no da recomendaciones sobre qué hacer si se detectan anomalías.

- Indica revisar efectividad a los 6 meses. No facilita orientación sobre posible interrupción del fármaco.

- Resta importancia a las conclusiones del MTA a 8 años sobre consumo de tóxicos y conductas transgresoras en edad adulta por alto porcentaje de interrupción de la medicación.

- Cree que se debe investigar más en la efectividad de la terapia conductual, la interrupción de la medicación y su timing, cuándo dejar la medicación y cómo.

- Hace hincapié en el consentimiento informado de calidad, incluyendo sospechas de problemas de crecimiento, y la implicación del niño en el tratamiento, comprensión de sus dificultades y responsabilidad en el manejo del trastorno. En el consentimiento informado incluye referencias a "niños mayores" pero no indica qué se puede hacer si el niño no desea el tratamiento farmacológico.

\section{GPC ESPAÑA, $2010(5)$}

-Elaborada por profesionales con lazos con la industria farmacéutica en sus dos terceras partes.

-Se basa en RS y Metanálisis ajenos, básicamente de otras GPC que considera de calidad, fundamentalmente la GPC NICE, única considerada muy recomendada y que cita explícitamente en las recomendaciones, y la SIGN -versión 2005-, recogiendo ECAs a iniciativa propia y añadiendo criterios basados en el consenso y la experiencia propia del equipo redactor.

-Sus recomendaciones son, a menudo, adaptaciones y glosas de las recomendaciones de otras GPC. En aspectos significativos y controvertidos sigue a una u otra, o a ninguna, sin dar explicaciones claras del motivo. Parece subyacer la opinión del equipo redactor.

-Para el metilfenidato recoge estudio MTA a 14 y 24 meses y 3 años.

-Considera el TDA/H como un trastorno de origen neurobiológico y de base genética.

- Recomienda terapia cognitivo conductual como tratamiento inicial en cuadros de sintomatología leve, repercusión funcional mínima, discrepancias sobre frecuencia e intensidad de síntomas entre padres o entre estos y profesores y diagnóstico incierto y en niños menores de cinco años (que quedan fuera del alcance de la guía). En la práctica no parece haber punto de corte puesto que se trata de casos muy leves y/o en los que no hay diagnóstico propiamente dicho.

- En todos los demás casos, subtipos TDA/H, edad y género del paciente, recomienda abordaje psicofarmacológico como primera opción.

- Considera igualmente efectivos el metilfenidato y la atomoxetina, a pesar de citar GPCs y ECAs que indican lo contrario. Ambos son de primera opción, con alusiones a comorbilidad para elegir uno u otro, preferencias de niño/ familia y, sobre todo, valoración posterior una vez iniciado el tratamiento sobre efectividad y efectos adversos. 
- Recomienda el tratamiento combinado para cuadros de intensidad moderada o grave, con muchas dudas sobre el valor añadido de las intervenciones psicológicas que, en cualquier caso, presenta como coadyuvantes a la potencialidad terapéutica del medicamento. Servirían para reducir la dosis del fármaco y aminorar las preocupaciones acerca del uso de la medicación en padres y niños.

- Considera efectivos a largo plazo tanto el metilfenidato (MTA 12 y 24 meses) como la atomoxetina (2 años). Corrige, desde un punto de vista más favorable, la interpretación de resultados del MTA a 3 años realizada por los autores del estudio.

- El tratamiento psicofarmacológico ha de mantenerse indefinidamente realizando valoraciones periódicas sobre su beneficio y efectos secundarios. No recomienda vacaciones terapéuticas salvo casos en los que el crecimiento esté afectado significativamente. Acepta la práctica de suspender tratamiento durante un breve periodo ( 1 o 2 semanas) para valorar persistencia de síntomas.

- En general, considera los efectos secundarios a corto plazo leves y manejables, y aconseja monitorización de FC, TA, crecimiento y peso. No presenta criterios operativos para toma de decisiones sobre continuidad del tratamiento si se detectan anomalías en la monitorización.

- Cita diversos estudios que recogen disminución de talla y peso concluyendo que, aunque no hay consenso, puede existir una leve disminución que se atenúa en el tiempo. Sugiere aporte nutricional.

- Niega riesgo de adicción a estimulantes y/o aumento del riesgo futuro de adicción al uso de sustancias. Atribuye carácter protector a la toma de estimulantes sobre el uso de sustancias en la edad adulta.

- Entre las líneas de investigación que propone de cara al futuro, recoge el estudio de beneficios o desventajas del uso a largo plazo de psicofármacos comparándolo con su interrupción a los 18 meses.

- Presenta un consentimiento informado de contenido tranquilizador y directivo respecto al uso de psicofármacos, minimizado, respecto al contenido de la propia guía, las referencias a afectos adversos y riesgos, y realizando afirmaciones rotundas y sin matices respecto al beneficio del uso de fármacos.

- Desde un punto de vista ético, recoge genéricamente la necesidad de contar con la opinión del menor maduro, y la posibilidad de recurrir a una autorización judicial en caso de especial conflictividad entre padres a la hora de iniciar un tratamiento ante el TDA/H, para poder aplicar el criterio de beneficencia para el menor.

GPC NHMRC, 2012, Australia (21)

-Realizada por el “ADHD Expert Working Group”, no explica cómo surge este grupo y si tiene o no conflictos de interés. -El TDA/H es un diagnóstico descriptivo con una etiología multifactorial.

-Recoge seguimiento MTA a seis y ocho años y el estudio RAINE.

-Cita las guías NICE y SIGN diciendo que no viene a sustituirlas sino a completarlas.

- Propone apoyo familiar y programa de entrenamiento parental si sospecha de TDA/H antes incluso de un diagnóstico formal por un especialista.

- Aconseja búsqueda de explicaciones no necesariamente médicas (evaluación psicosocial, familiar, cultural, significado de síntomas teniendo en cuenta edad, desarrollo y expectativas del niño).

- Salvo en los menores de 7 años, para quienes se recomienda tratamiento no farmacológico en primera instancia, la primera opción puede ser psicológica, farmacológica, o educacional, solas o en combinación. Depende de la evaluación clínica y el plan de tratamiento, considerando en primer lugar aquellos factores que afecten más severamente al funcionamiento del menor.

- Respecto a intervenciones psicológicas, para los menores de 7 años recomienda terapia psicológica, conductual y familiar. Para niños entre 7-12, las mismas más la cognitivo conductual. Para adolescentes, terapia cognitivo conductual.

- Considera efectivos el metilfenidato y la dexamfetamina. No recoge la atomoxetina por motivos regulatorios en Australia.

- Considera más eficaces los estimulantes y la terapia combinada que las intervenciones psicosociales y conductuales solas (MTA a 24 meses).

- Cree que no esta demostrada la eficacia y seguridad a largo plazo, más de tres años, de los estimulantes, resaltando que hay datos contradictorios (MTA a 3 y 8 años) y que no está contrastada su superioridad sobre procedimientos no psicofarmacológicos a largo plazo (MTA a 8 años).

- Considera que los efectos indeseados de los fármacos son en general menores: TA, problemas de sueño, problemas de crecimiento.

- Llama, no obstante, a monitorizar: FC, TA, peso y altura, considerando percentiles.

- Recomienda supervisar el crecimiento, el desarrollo de la pubertad y la posible aparición de efectos psicológicos indeseados.

- Recomienda continuar el tratamiento si se demuestran beneficios y en ausencia de efectos adversos "inaceptables" (no los describe). Habla de "señales" para detener el tratamiento (no las explicita).

- Si se considera que no se necesitan estimulantes plantea interrupción del tratamiento como mínimo durante varias semanas y en un momento adecuado (no lo explicita).

- Aboga por un consentimiento informado de calidad, buscando planes alternativos si el fármaco no es aceptado por el menor o sus tutores. 
ORIGINALES Y REVISIONES

Respecto a la duración del tratamiento, las GPC indican que ha de ser indefinido mientras se muestre beneficioso, evaluando regularmente efectos adversos y beneficiosos, y no recomiendan vacaciones terapéuticas, indicando en todo caso la interrupción por breves períodos de tiempo para valorar la necesidad o no de seguir con el tratamiento. Se desmarca la guía australiana que sí parece tener en cuenta el posible efecto sintomático de discontinuidad derivado de la interrupción brusca en la toma del fármaco (54), recomendando interrupción "como mínimo de varias semanas" y "en el momento adecuado" (que no precisa) (21). Sorprendentemente, la guía NICE propone como línea de investigación futura la interrupción del fármaco a los 18 meses puesto que "se prescribe sobre periodos largos de años sin evidencia significativa", sugerencia que también recoge la guía española $(3,5)$.

Respecto a la interrupción por efectos adversos, la GPC NICE facilita un punto de corte en lo que se refiere a cambios en TA y FC para derivar al médico de familia (3). Nada dice sobre qué hacer en caso de hallazgo de alteraciones en el crecimiento, peso y talla. No obstante, se puede rastrear las preocupaciones en torno a estos temas a la lectura del texto completo de las guías -p.ej. la preocupación respecto al desarrollo madurativo cerebral en niños en edad preescolar- y en el consentimiento informado que, en general, salvo la española, se formulan en términos de consentimiento informado de calidad, facilitando información detallada incluso sobre aspectos controvertidos y terapias alternativas en caso de rechazo de padres y niños $(34,54)$.

\subsection{La perspectiva clínica actual}

El consentimiento informado es un punto central en la ética asistencial construida sobre el principio de autonomía, y otros principios éticos como "tratar como querrías ser tratado". La propuesta que facilita la guía española es pobre y directiva a favor de la utilización de fármacos. Otros textos de consentimiento, mejor orientados, adolecen del defecto de hacer afirmaciones rotundas no contrastadas por los hechos, como p.ej. que en el TDA/H el metilfenidato trae una mejoría al 70\% de los pacientes (132). El texto de consentimiento informado resulta así un eslabón de un relato habitual más amplio que afirma que el TDA/H es un trastorno con mala evolución sin el fármaco que, aun sin revertir la condición del TDA/H, lo palia de forma eficaz, y que no tiene alternativa.

Lo que dicen los datos que hemos revisado es que nunca se ha demostrado que funcione el tratamiento farmacológico en ensayos controlados mas allá de cuatro semanas, que hay un declive de la mejoría cuando se mantiene el tratamiento a largo plazo, que con los años los niños tratados con fármacos tendrán peor funcionamiento y necesitarán más apoyo escolar en el futuro que los que nunca fueron medicados, que a medio plazo no hay diferencia entre la medicación y una intervención psicológica no muy sofisticada, que puede producirse daños preocupantes por la toma continuada del fármaco y que el riesgo es mayor con la exposición temprana al mismo. 
La mejoría en la atención a corto plazo se da en los pacientes diagnosticados de TDA/H así como en los demás menores y adultos de forma generalizada con el uso de estimulantes, probablemente en más del 70\%. La mejoría, en el mejor de los supuestos, parece darse sólo durante unos meses y en dos aspectos: parcialmente en la atención y en el movimiento. No hay prueba alguna de que haya mejoría en impulsividad, comportamiento disruptivo, relaciones sociales y autoestima, disminución de riesgos futuros, etc.

En definitiva, el valor del fármaco es su beneficio limitado a corto plazo. Puede ser útil estratégicamente como ayuda en un momento determinado, o como un recurso para casos complicados que permita ganar tiempo para que el entorno se organice con el objetivo de proporcionar al menor una ayuda, en términos no muy diferentes a los que utiliza la Guía NICE para justificar el interés del tratamiento combinado en casos graves. En cualquier caso, el uso de medicación debe de ser considerado como una herramienta de último recurso (133). Una GPC basada en lo que se conoce actualmente sería muy diferente a las GPC estudiadas.

Proponemos en la Tabla 4 un breve resumen de evidencias y recomendaciones sobre las que podría construirse una GPC actualizada.

De los niños tratados farmacológicamente en USA por TDA/H quizás sólo en el $10 \%$ de los casos se podría considerar el medicamento $(14,134)$. Es posible que no sea muy diferente en España.

TABLA 4:

Bases de evidencia científica y recomendaciones clínicas para una GPC actualizada

\begin{abstract}
-No hay prueba alguna de la eficacia a medio y largo plazo de los psicofármacos en el tratamiento del TDA/H, ni de su superioridad sobre los procedimientos no psicofarmacológicos, y sí de sus potenciales perjuicios y riesgos, incluso sobre la evolución de los propios síntomas nucleares del TDA/H.

-Ni el metilfenidato ni la atomoxetina pueden ser la primera opción o el abordaje principal del tratamiento de un menor diagnosticado de TDA/H cualquiera que sea su edad, intensidad y subtipo.

-La utilización de fármacos puede tener su lugar y sentido estratégico en el plan de tratamiento, sobre periodos limitados y, en general, breves de tiempo

-La ayuda real a medio y largo plazo ha de obtenerse desde otros procedimientos clínicos y ámbitos de actuación.
\end{abstract}

1. Ante la sospecha de TDA/H, y previamente a cualquier tratamiento, se necesita una evaluación que establezca que los signos no se entienden mejor considerando otros factores y que son incompatibles con el nivel de desarrollo del niño.

2. Si un niño recibe un diagnóstico de TDA/H en preescolar, los estimulantes deben evitarse, ya que puede resolverse en uno o dos años por si mismo.

3. Las intervenciones psicológicas deben utilizarse primero, dado que su eficacia es comparable al fármaco y no conlleva sus riesgos biológicos. Han de ser complementadas con intervenciones educativas y, en su caso, sociales.

4. Si el niño no ha respondido adecuadamente a los 6 meses en un tratamiento psicólogico se puede probar con otro de diferente orientación o formato, antes de considerar la opción farmacológica.

5. Los fármacos han de ser utilizados como herramientas de último recurso y sobre períodos de tiempo limitados y breves.

6. Las intervenciones psicológicas deben de utilizarse siempre, aún cuando se prescriban fármacos, para incrementar la competencia del niño y su entorno en el manejo de los problemas (Estas seis recomendaciones han sido adaptadas de LeFever (19), y Diller (134)). 
ORIGINALES Y REVISIONES

Finalizaremos con la declaración al diario de The Guardian del Dr. William Pelham, investigador del estudio MTA, cuando los datos del seguimiento a los 3 años del MTA ya habían trascendido a los medios: "Creo que exageramos el impacto beneficioso de la medicación en el primer estudio. Pensamos que los niños medicados durante más tiempo tendrían mejores resultados. Pero no ocurrió así. Los niños crecieron menos de forma sensible, no crecieron como el resto de los niños ni en talla ni en peso. Y segundo, no hubo ningún efecto beneficioso, ninguno. A corto plazo, (la medicación) ayudó a los niños a comportarse mejor, pero no a largo plazo. Y esta información debería darse de forma clara a los padres" (135).

BIBLIOGRAFÍA:

(1) Hallowell EM, Ratey JJ. TDA: controlando la hiperactividad. barcelona: Paidós (1994) 2001.

(2) Storebø OJ, Rosendal S, Skoog M, Groth C, Bille T, Buch Rasmussen K, et al. Methylphenidate for attention deficit hyperactivity disorder (ADHD) in children and adolescents (Protocol). The Cochrane Library, Issue 5, 2012.

(3) GPC NICE 2009 - National Collaborating Centre for Mental Health Royal College of Psychiatrists' Research and Training Unit. Attention deficit hyperactivity disorder: diagnosis and management of ADHD in children, young people and adults. National clinical practice guideline number 72. London: The British Psychological Society and The Royal College of Psychiatrists; 2009. [Consultado 12-12-2012]. Disponible en: http://www.nice.org.uk/nicemedia/ live/12061/42060/42060.pdf

(4) GPC SIGN 2009 - Scottish Intercollegiate Guidelines Network (SIGN). Management of attention deficit and hyperkinetic disorders in children and young people: A national clinical guideline 112. Edinburgh: SIGN; 2009. [Consultado 12-12-2012] Disponible en: http://www.sign.ac.uk/pdf/ sign 112.pdf

(5) GPC ESPAÑA 2010 - Grupo de trabajo de la Guía de Práctica Clínica sobre el Trastorno por Déficit de Atención con Hiperactividad (TDA/H) en Niños y Adolescentes. Fundació Sant Joan de Déu, coordinador. Guía de Práctica Clínica sobre el Trastorno por Déficit de Atención con Hiperactividad (TDA/H) en Niños y Adolescentes. Plan de Calidad para el Sistema Nacional de Salud del Ministerio de Sanidad, Política Social e Igualdad. Agència d'Informació, Avaluació i Qualitat (AIAQS) de Cataluña; 2010. Guías de Práctica Clínica en el SNS: AATRM No 2007/18 [Consultado 30-1-2013] Disponible en: http://www.gencat.cat/salut/depsan/units/aatrm/pdf/gpc tdah_hiperactividad_aiaqs2010_compl.pdf

(6) Mayes R, Bagwell C, Erkulwater J. Medicating children. ADHD and pediatric mental health. USA: Harvard University Press, 2009.

(7) Timimi S. Mis-Understanding ADHD: The complete guide for parents to alternatives to drugs. USA: AutorHouse, 2007.

(8) Kean B. ADHD in Australia: The emergence of globalization. En: Timimi S, Leo J. editors. Rethinking ADHD. From brain to culture. UK: Palgrave and Macmillan 2009; p. 169-97. 
(9) Grupo Consenso TDAH Multidisciplinar. Consenso multidisciplinar en TDA/H: infancia , adolescencia y adultos. España: 2005. [Consultado 13-1-2013] Disponible en: http:// anadahialava.files.wordpress.com/2010/12/consenso-multidisciplinar.pdf

(10) Lasa-Zulueta A, Jorquera-Cuevas C. Evaluación de la situación asistencial y recomendaciones terapéuticas en el trastorno por déficit de atención e hiperactividad. Plan de Calidad para el Sistema Nacional de Salud del Ministerio de Sanidad y Política Social. Agencia de Evaluación de Tecnologías Sanitarias del País Vasco 2009. Informes de Evaluación de Tecnologías Sanitarias:

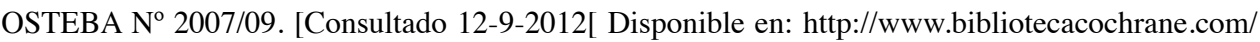
BCPGetDocument.asp?SessionID=\%205477340\&DocumentID=AEV000071

(11) Pandah. Rev. Comunidad TDA/H. 2011. [Consultado 10-12-2012] Disponible en: http:// www.comunidad-tdah.com/noticia/el-proyecto-pandah-aborda-las-vertientes-sanitaria-economica-ysociologica-del-tdah

(12) Timimi S. The politics of Attention Deficit Hyperactivity Disorder (ADHD). En: Timimi S, Maitra B, editors. Critical voices in child and adolescence mental health. UK: Free Association Books, 2005; p. 163-91.

(13) Vallée M. Biomedicalizing mentall illness: the case of attention deficit disorder. En: Mukherjea N, Editor. Understanding emerging epidemics: social and political approaches. USA: Emerald Books, 2010; p. 281-231.

(14) Horwitz A. Pharmaceuticals and the medication of social life. En: Light DW. Editor. The risks of prescription drugs. New York: Columbia University Press, 2010; p. 92-115.

(15) Barkley RA. Niños hiperactivos: como comprender y atender sus necesidades especiales. Barcelona: Paidos, 1999.

(16) Barkley RA, Cook EH, Diamond A, Zametkin A, Thapar A, Teeter A, et al. International Consensus Statement on ADHD January 2002. Clinical Child and Family Psychology Review, Vol. 5, No. 2, June 2002, 89-111.

(17) Global ADHD Working Group Global consensus on ADHD/HKD. Eur Child Adolesc Psychiatry 2005, 14:127-137.

(18) Montañés-Rada F, Gastaminza-Pérez X, Catalá MA, Ruiz-Sanz F, Ruiz-Lázaro PM, Herreros-Rodríguez O, et al. Grupo de Especial Interés en el TDAH (GEITDAH). Consenso del GEITDAH sobre el trastorno por déficit de atención/hiperactividad. Rev Neurol 2010; 51: 633-7.

(19) LeFever GB, Arcona AP, Antonuccio DO. ADHD among American Schoolchildren. Evidence of Overdiagnosis and Overuse of Medication. SRMHP Home / Spring Summer 2003 Volume 2 Number 1. [Consultado 3-10-2012] Disponible en: http://www.srmhp.org/0201/adhd.html

(20) Jackson GE. The case against the stimulants. En: Timimi S, Leo J. Editors Rethinking ADHD. From brain to culture. UK: Palgrave and Macmillan. 2009; p. 255-86.

(21) GPC NHMRC 2012. National Health and Medical Research Council, Australia. Clinical Practice Points on the diagnosis, assessment and management of Attention Deficit Hyperactivity Disorder in children and adolescents. Commonwealth of Australia. 2012. [Consultado 12-12-2012] Disponible en: http://www.nhmrc.gov.au/_files_nhmrc/publications/attachments/mh26_adhd_ cpp_2012_120903.pdf

(22) Methylphenidate: abuse in Europe Prescrire International 2013, 22 (135):47.

(23) MTA Cooperative Group. A 14-month randomized clinical trial of treatment strategies for attention-deficit/hyperactivity disorder. Arch Gen Psychiatry. 1999 Dec;56(12):1073-86. 
(24) Furman L. ADHD: What Do We Really Know? En: Timimi S, Leo J. Editors Rethinking ADHD. From brain to culture. UK: Palgrave and Macmillan. 2009; p. 21-57.

(25) King S, Griffin S, Hodges Z, Weatherly H, Asseburg C, Richardson G, et al. A systematic review and economic model of the effectiveness and cost-effectiveness of methylphenidate, dexamfetamine and atomoxetine for the treatment of attention deficit hyperactivity disorder in children and adolescents Health Technology Assessment 2006; Vol. 10: No. 23.

(26) Drug ERPO 2011 - McDonagh MS, Peterson K, Dana T, \& Takhurta S. Drug Effectiveness Review Project at Oregon State University. Pharmacologic Treatments for Attention Deficit Hyperactivity Disorder Final Update 4 Report 2011. [Consultado 31-8-2012)] Disponible en: www.ohsu.edu/drugeffectiveness/reports/final.cfm

(27) Timimi S, Leo J. editors. Rethinking ADHD. From brain to culture. UK: Plagrave and Macmillan, 2009.

(28) Jensen P, Arnold E, Swanson J. Vitiello B, Abikoff H, Greenhill LL, et al. 3-Year Followup of the NIMH MTA Study. J. Am. Acad. Child Adolesc.Psychiatry 46:8, August 2007, p. 989-1002.

(29) Molina B, Flory K, Hinshaw SP, Greiner Ar, Arnold E, Swanson J, et al. Delinquent Behavior and Emerging Substance Use in the MTA at 36 Months: Prevalence, Course, and Treatment Effects. J. Am. Acad. Child Adolesc. Psychiatry 46:8, August 2007, 1028-40.

(30) Molina B, Hinshaw SP, Swanson JM, Arnold LE, Vitiello B, Jensen PS, et al. MTA at 8 Years: Prospective Follow-up of Children Treated for Combine Type ADHD in a Multisite Study. J. Am. Acad. Child Adolesc. Psychiatry 2009, 48:5, 484-500.

(31) Hazell PL. 8-Year Follow-up of the MTA Sample. J. Am. Acad. Child Adolesc. Psychiatry 2009, 48:5, 461-462.

(32) Myttas N. Clinical and Neurological Differentiation in ADHD. ADHD in Practice, $2009,1,6,22-23$.

(33) RAINE 2011 - Smith G, Jongeling B, Hartmann P, Russell C, Landau L. Raine ADHD Study: Long-term outcomes associated with stimulant medication in the treatment of ADHD in children. Western Australian Department of Health, 2011. [Consultado 12-12-2012] Disponible en: http://www.health.wa.gov.au/publications/documents/MICADHD_Raine_ADHD_Study_ report_022010.pdf

(34) Jackson GE. Rethinking Psychiatric Drugs: A Guide for Informed Consent. USA: AuthorHouse 2005.

(35) Jackson GE. Drug-Induced dementia. A perfect crime. USA: AuthorHouse 2009.

(36) Mattison DR, Plant TM, Hui-Min Lin, Hung-Chia Chen, Chen JJ, Twaddle NC, et al. Pubertal delay in male nonhuman primates (Macaca mulatta) treated with methylphenidate. Proc Natl Acad Sci U S A. 2011 Sep 27;108(39):16301-6. [Consultado 10-1-2013] Disponible en: http://www. pnas.org/content/early/2011/09/12/1102187108.full.pdf

(37) Urban KR, Waterhouse BD, Gao WJ.Distinct Age-Dependent Effects of Methylphenidate on Developing and Adult Prefrontal Neurons Biol Psychiatry. 2012 Nov 15;72(10):880-8.

(38) Consorcio AGREE. AGREE II. Instrumento para la evaluación de guías de práctica clínica. (Appraisal of Guidelines for Research and Evaluation). [Consultado 12-12-2012] Disponible traducción española en: http://www.agreetrust.org/resource-centre/agree-ii-translations/ 
(39) Jadad AR, Moore RA, Carroll D, Jenkinson C, Reynolds DJ, Gavaghan DJ, McQuay HJ. Assessing the quality of reports of randomized clinical trials: is blinding necessary? Control Clin Trials. 1996 Feb;17(1):1-12.

(40) MTA Cooperative Group. National Institute of Mental Health. Multimodal Treatment Study of ADHD follow-up: 24-month outcomes of treatment strategies for attention-deficit/ hyperactivity disorder. Pediatrics. 2004 Apr;113(4):754-61.

(41) Swanson JM, Elliott GR, Greenhill LL, Wigal T, Arnold LE, Vitiello B, et al. Effects of stimulant medication on growth rates across 3 years in the MTA follow-up. J Am Acad Child Adolesc Psychiatry. 2007 Aug;46 (8):1015-27.

(42) Gillberg C, Melander H, von Knorring AL, Janols LO, Thernlund G, Hägglöf B, et al. Long-term stimulant treatment of children with attention-deficit hyperactivity disorder symptoms. A randomized, doubleblind, placebo-controlled trial. Arch Gen Psychiatry. 1997;54(9):857-64.

(43) Lambert N, Hartsough CS. Prospective Study of Tobacco Smoking and Substance Dependencies among Samples of ADHD and Non-ADHD Participants Journal of Learning Disabilities Volume 31, Number 6, November/December 1998, 533-544.

(44) Lambert N. The contribution of childhood ADHD, conduct problems, and stimulant treatment to adolescent and adult tobacco and psychoactive substance abuse. Ethical Hum Psychol Psychiatry. 7(3): 2005. 197-221.

(45) Charach A, Ickowicz A, Schachar R. Stimulant treatment over five years: adherence, effectiveness, and adverse effects. J Am Acad Child Adolesc Psychiatry. 2004;43(5):559-67.

(46) Barbaresi WJ, Katusic SK, Colligan RC, Weaver AL, Leibson CL, Jacobsen SJ. Longterm stimulant medication treatment of attention-deficit/hyperactivity disorder: results from a population-based study. J Dev Behav Pediatr. 2006;27(1):1-10.

(47) Kratochvil CJ, Wilens TE, Greenhill LL, Gao H, Baker KD, Feldman PD, et al. Effects of long-term atomoxetine treatment for young children with attention-deficit/hyperactivity disorder. $\mathrm{J}$ Am Acad Child Adolesc Psychiatry. Aug 2006; 45(8):919-27.

(48) Wilens TE, Newcorn JH, Kratochvil CJ, Gao H, Thomason CK, Rogers AK et al. Longterm atomoxetine treatment in adolescents with ADHD attention-deficit/hyperactivity disorder. $\mathrm{J}$ Pediatr. Jul 2006;149(1):112-9.

(49) GPC AHRQ 2011 - Charach A, Dashti B, Carson P, Booker L, Lim CG, Lillie E. \& al. Attention Deficit Hyperactivity Disorder: Effectiveness of Treatment in At-Risk Preschoolers; LongTerm Effectiveness in All Ages; and Variability in Prevalence, Diagnosis, and Treatment. Comparative Effectiveness Review No. 44. (Prepared by the McMaster University Evidence-based Practice Center under Contract No. MME2202 290-02- 0020.) AHRQ Publication No. 12-EHC003-EF. Rockville, MD: Agency for Healthcare Research and Quality. October 2011. [Consultado 13-1-2013] Disponible en: http://effectivehealthcare.ahrq.gov/ehc/products/191/818/CER44-ADHD_20111021.pdf

(50) GPC AAP 2011 - American Academy of Pediatrics. Management steering committee on quality improvement and Subcommittee on attention-deficit/hyperactivity disorder. ADHD: Clinical Practice Guideline for the Diagnosis, Evaluation, and Treatment of Attention-Deficit/Hyperactivity Disorder in Children and Adolescents. Pediatrics; 2011. 128(5):1007-1022.

(51) Schachter HM, Ba' Pham, King J, Langford S, Moher D. How efficacious and safe is short acting methylphenidate for the treatment of attention-deficit disorder in children and adolescents A meta-analysis. CMAJ. 2001 Nov 27;165(11):1475-88. 
ORIGINALES Y REVISIONES

(52) Long-Term Open Label Atomoxetine Study. ClinicalTrials.gov Identifier: NCT00190684. [Consultado el 30-10-2012] Disponible en http://clinicaltrials.gov/ct2/show/record/ NCT00190684

(53) Double D, Timimi S. National Institute for Health \& Clinical Excellence Attention Deficit Hyperactivity Disorder. Stakeholder Comments. [Consultado el 12-12-2012] Disponible en http://www.critical.freeuk.com/ ADHDConsComments.doc. Réplica de NICE en ADHDreply.htm (Comprobado el 12-12-2012).

(54) Moncrieff J. The myth of chemical cure. A critique of psychiatric drug treatment. 2nd Edition. UK: Palgrave, 2009.

(55) Moncrieff J. Straight talking introduction to psychiatric drugs. UK: PCCS-books, 2009.

(56) Timimi S. Straight talking introduction to Children's Mental Health Problems. UK: PCCS-books, 2009.

(57) Whitaker R. Anatomy of an Epidemic: Magic Bullets, Psychiatric Drugs, and the Astonishing Rise of Mental Illness in America New York: Crown Publishers 2010.

(58) Jackson G. Letter about a Law Project For Pscyhiatric Rights State vs. State of Alaska 2009. [Consultado 2-2-2013C] Disponible en: http://psychrights.org/research/Digest/ADHD/090331 GraceJacksonAffidaviOnWeiszandJensent.pdf

(59) Stahl S. Stahl's Esssential Psychopharmacology. Neurosientific Basis and Practical Applications Third Edition, Cambridge University Press 2008.

(60) Anatomical Therapeutic Chemical Classification System; N06BA Centrally acting sympathomimetics. WHO, 2012 [Consultado 15-1-2013] Disponible en: http://www.whocc.no/atc_ ddd_index/?code=N06BA

(61) Abikoff HB, Vitiello B, Riddle MA, Cunningham C, Greenhill LL, Swanson JM, et al. Methylphenidate Effects on Functional Outcomes in the Preschoolers with Attention-Deficit/ Hyperactivity Disorder Treatment Study (PATS). J Child Adolesc Psychopharmacol. 2007 Oct;17(5):581-92.

(62) Loro-López M, Quintero J, García-Campos N, Jiménez-Gómez B, Pando F, VarelaCasal P, et al. Actualización en el tratamiento del trastorno por déficit de atención/hiperactividad. Rev Neurol 2009; 49 (5): 257-264.

(63) Breggin PR. The NIMH multimodal study of treatment for attention-deficit/hyperactivity disorder: A critical analisis. International Journal of Risk \& Safety in Medicine 2000, 13 15-22.

(64) Pelham WE, Gnagy EM, Greiner AR, Hoza B, Hinshaw SP, Swanson JM, et al. Behavioral versus Behavioral and Pharmacological Treatment in ADHD Children Attending a Summer Treatment Program. Journal of Abnormal Child Psychology 3000, Volume 28, Number 6, 507-525.

(65) Swanson JM, Hinshaw SP, Arnold LE, Gibbons RD, Marcus S, Hur K, et al. Secondary evaluations of MTA 36-month outcomes: propensity score and growth mixture model analyses. J Am Acad Child Adolesc Psychiatry. 2007 Aug;46(8):1003-14.

(66) Pappadopulos E, Jensen PS, Chait AR, Arnold E, Swanson JM, Greenhill LL. et al Medication Adherence in the MTA: Saliva Methylphenidate Samples Versus Parent Report and Mediating Effect of Concomitant Behavioral Treatment J Am Acad Child Adolesc Psychiatry. 2009 May; 48(5):501-10. 
(67) Whitley M. Speed up \& sit still. The controversias of ADHD Diagnosis and treatment. Australia: UWA Publishing, 2010.

(68) Whitley, M. One year on from the Raine Study ADHD Medication Review - Will the analysis of this unique long term data source continue and if so can we trust those doing the analysis?, 2011, [Consultado 19-1-2013] Disponible en: http://speedupsitstill.com/raine-study-review-oneyear-on

(69) Cohen D, Hughes Sh, \& Jacobs DJ. The Deficiencies of Drug Treatment Research: The Case of Strattera ${ }^{\mathrm{TM}}$. En: Timimi S, Leo J. Editors Rethinking ADHD. From brain to culture. UK: Palgrave and Macmillan. 2009; p. 313-33.

(70) Stolerman IP. Editor. Encyclopedia of Psychopharmacology. Springe-Verlag Berlin Heidelberg: 2010.

(71) Azanza JR. Guía práctica de Farmacología del Sistema Nervioso Central. Gumbau R.G. Barcelona: 2011.

(72) Velásquez-Tirado JD, Peña JA. Evidencia actual sobre la atomoxetina. Alternativa terapéutica para el trastorno por déficit de atención e hiperactividad Rev Neurol 2005; 41 (8): 493500 .

(73) Newcorn JH, Sutton VK, Weiss MD, Sumner CR. Clinical Responses to Atomoxetine in Attention-Deficit/Hyperactivity Disorder: The Integrated Data Exploratory Analysis (IDEA) Study. J Am Acad Child Adolesc Psychiatry. 2009 May;48(5):511-8

(74) Manga D, González H, Fournier C. Trastornos por déficit de atención e hiperactividad. En: Belloch A. Sandin B, Ramos F. Editors. Manual de psicopatología. Edición revisada. Volumen II. Madrid: McGraw Hill. 2009.

(75) Hyman S. Initiation and adaptation: A paradigm for understanding psychotropic drug action. Am J Psychiatry 1996,153,151-161.

(76) Vles JS, Feron FJ, Hendriksen JG, Jolles J, van Kroonenburgh MJ, Weber WE. Methylphenidate down-regulates the dopamine receptor and transporter system in children with attention deficit hyperkinetic disorder (ADHD). Neuropediatrics 2003 Apr;34(2):77-80.

(77) Ferreira R, Bassi GS, Cabral A, Nobre MJ. Withdrawal from methylphenidate increases neural reactivity of dorsal midbrain. Neurosci Res. 2010 Dec;68(4):290-300.

(78) Nolan EE, Gadow KD, Sprafkin J. Stimulant medication withdrawal during long-term therapy in children with comorbid attention-deficit hyperactivity disorder and chronic multiple tic disorder. Pediatrics. 1999 Apr;103(4 Pt 1):730-7.

(79) Gadow KD, Sverd J, Sprafkin J, Nolan EE, Grossman S. Long-term methylphenidate therapy in children with comorbid attention-deficit hyperactivity disorder and chronic multiple tic disorder. Arch Gen Psychiatry. 1999 Apr;56(4):330-6.

(80) Arnsten AF, Dudley AG. Methylphenidate improves prefrontal cortical cognitive function through $\alpha 2$ adrenoceptor and dopamine D1 receptor actions: Relevance to therapeutic effects in Attention Deficit Hyperactivity Disorder. Behavioral and Brain Functions, 2005,Vol 1, Apr, Article 2. doi: 10.1186/1744-9081-1-2.

(81) Breggin PR. Talking back to Ritalin. What doctors aren't telling you about stimulants and ADHD. Cambridge, MA: Perseus Publishing. 2001.

(82) Gardner BK, Sheppard DM, Efron D. The impact of stimulants on a clinical measure of attention in children with ADHD. Child Neuropsychol. 2008 Mar;14(2):171-86. 
(83) Schmidt Neven R, Anderson V, Godber T. Rethinking ADHD: Integrated approaches to helping children at home and at school. Australia: Allen \& Unwin 2002.

(84) Eichlseder W. Ten years of experience with 1,000 hyperactive children in a private practice. Pediatrics. 1985, 76(2):176-84.

(85) Sleator EK, Ullmann RK, von Neumann A. How do hyperactive children feel about taking stimulants and will they tell the doctor? Clin Pediatr (Phila) 1982,21(8):474-9.

(86) Swanson J. Compliance with stimulants for attention-deficit/hyperactivity disorder: issues and approaches for improvement. CNS Drugs. 2003; 17(2):117-31.

(87) Miller AR, Lalonde CE, McGrail KM. Children's Persistence With Methylphenidate Therapy A Population-Based Study Can J Psychiatry. 2004 Nov;49(11):761-8.

(88) Gould MS, Walsh BT, Munfakh JL, Kleinman M, Duan N, Olfson M, et al. Sudden Death and Use of Stimulant Medications in Youths Am J Psychiatry 2009 Sep;166(9):992-1001.

(89) Sans Fitó A. ¿Por qué me cuesta tanto aprender? Trastornos del aprendizaje $3^{\text {a }}$ Edición. Barcelona: Edebé, 2010.

(90) Lee Negrao B, Viljoen M. Stimulants and growth in children with attention-deficit/ hyperactivity disorder Med Hypotheses. Jul 2011;77(1):21-8.

(91) Méthylphénidate: retards de croissance staturo-pondérale Rev. Prescrire 2011;31 (328):108-109.

(92) Ptacek R, Kuzelova H, Paclt I. Effect of stimulants on growth of ADHD children: a critical review Activitas Nervosa Superior 2009,51:4,140-146.

(93) Kilgore BS, Dickinson MA, Burnett CR, Lee J, Schedewie HK, Elder MJ. Alterations in cartilage metabolism by neurostimulant drugs. J Pediatr.1979;94:542-45.

(94) O’Brien LM, Ivanenko A, McLaughlin Crabtree V, Holbrook CR, Leigh Bruner J, Klaus CJ. Sleep Disturbances in Children with Attention Deficit Hyperactivity Disorder. Pediatric Research Vol. 54,2,2003.

(95) Faraone SV, Glatt SJ, Bukstein OJ, Lopez FA, Arnold LE, Findling RL. Effects of once-daily oral and transdermal methylphenidate on sleep behavior of children with ADHD. J Atten Disord.2009; 12(4):308-15.

(96) Gau SS, Chiang HL. Sleep Problems and Disorders among Adolescents with Persistent and Subthreshold Attention-deficit/Hyperactivity Disorders Sleep 2009,May;32(5):671-9.

(97) Touchette E, Côté SM, Petit D, Liu X, Boivin M, Falissard B, et al. Short Nighttime SleepDuration and Hyperactivity Trajectories in Early Childhood. Pediatrics. 2009 Nov;124(5):e985-93.

(98) Sangal RB, Owens J, Allen AJ, Sutton V, Schuh K \& Kelsey D. Effects of Atomoxetine and Methylphenidate on Sleep in Children With ADHD. Sleep. 2006 Dec;29(12):1573-85.

(99) Wigal T, Greenhill L, Chuang S, McGough J, Vitiello B, Skrobala A, et al. Safety and tolerability of methylphenidate in preschool children with ADHD. J Am Acad Child Adolesc Psychiatry. 2006 Nov;45(11):1294-303.

(100) Antle MC, van Diepen HC, Deboer T, Pedram P, Pereira RR, Meijer JH. Methylphenidate modifies the motion of the circadian clock. Neuropsychopharmacology. 2012 Oct;37(11):2446-55.

(101) Carlezon WA Jr, Mague SD, Andersen SL. Enduring behavioral effects of early exposure to methylphenidate in rats. Biol Psychiatry. 2003 Dec 15;54(12):1330-7.

(102) Vendruscolo LF, Izídio GS, Takahashi RN, Ramos A. Chronic methylphenidate treatment during adolescence increases anxiety-related behaviors and ethanol drinking in adult spontaneously hypertensive rats. Behavioural Pharmacology 2008, 19:21-27. 
(103) Fusar-Poli P, Rubia K, Rossi G, Sartori G, Balottin U. Striatal Dopamine Transporter Alterations in ADHD: Pathophysiology or Adaptation to Psychostimulants? A Meta-Analysis. Am J Psychiatry 169:3, March 2012.

(104) Spencer TS, Madras BK, Fischman AJ, Krause J, Fouger CL. Striatal Dopamine Transporter Binding in Adults With ADHD [carta]. Am J Psychiatry 169:6, June 2012 p 665.

(105) Fusar-Poli P. Response to Spencer et al [carta]. Am J Psychiatry 2012;169:666-666.

(106) Castellanos FX, Lee PP, Sharp W, Jeffries NO, Greenstein DK, Clasen LS, et al. Developmental trajectories of brain volume abnormalities in children and adolescents with attentiondeficit/hyperactivity disorder JAMA. 2002 Oct 9;288(14):1740-8.

(107) Mackie S, Shaw P, Lenroot R, Pierson R, Greenstein DK, Nugent TF 3rd, et al. Cerebellar development and clinical outcome in attention deficit hyperactivity disorder Am J Psychiatry. 2007 Apr;164(4):647-55.

(108) Shaw P, Eckstrand K, Sharp W, Blumenthal J, Lerch JP, Greenstein D et al. Attentiondeficit/hyperactivity disorder is characterized by a delay in cortical maturation Proc Natl Acad Sci U S A. 2007 December 4; 104(49): 19649-19654.

(109) Shaw P, Lerch J, Greenstein D, Sharp W, Clasen L, Evans A, et al. Longitudinal mapping of cortical thickness and clinical outcome in children and adolescents with attention-deficit/ hyperactivity disorder Arch Gen Psychiatry. 2006 May;63(5):540-9.

(110) Shaw P, Sharp WS, Morrison M, Eckstrand K, Greenstein DK, Clasen LS, et al. Psychostimulant treatment and the developing cortex in attention deficit hyperactivity disorder Am J Psychiatry 2009 Jan;166(1):58-63.

(111) Elkins IJ, McGue M, Iacono WG. Prospective Effects of Attention-Deficit/Hyperactivity Disorder, Conduct Disorder, and Sex on Adolescent Substance Use and Abuse Arch Gen Psychiatry 2007 Oct;64(10):1145-52.

(112) Lipkin PH, Goldstein IJ \& Adesman AR. Tics and dyskinesias associated with stimulant treatment in attention-deficit hyperactivity disorder. Arch Pediatr Adolesc Med. 1994,148(8):859-61.

(113) Varley CK, Vincent J, Varley P \& Calderon R. Emergence of tics in children with attention deficit hyperactivity disorder treated with stimulant medications. Compr Psychiatry. 2001, 42(3):228-33.

(114) Pidsosny IC, Virani A. Pediatric Psychopharmacology Update: Psychostimulants and Tics Past, Present and Future J Can Acad Child Adolesc Psychiatry 15:2 May 2006; 84-86.

(115) Pringsheim T, Steeves T. Pharmacological treatment for Attention Deficit Hyperactivity Disorder (ADHD) in children with comorbid tic disorders. Cochrane Database of Systematic Reviews 2011, Issue 4.

(116) Cherland, E. \& Fitzpatrick, R. Psychotic side effects of stimulants: A 5 year review. Can J Psychiatry 1999, 44:81-813.

(117) Biederman J, Faraone S, Mick E, Wozniak J, Chen L, Ouellette C. \& al. Attention-deficit hyperactivity disorder and juvenile mania: an overlooked comorbidity? J Am Acad Child Adolesc Psychiatry. 1996, 35: 997-1008.

(118) Abad I,Ariz MJ,Azparren A, Erviti J.,Garjón J, Gorricho, J,LópezA, Montoya R, Moreno M, Muruzábal L. Servicio Navarro de Salud Atomoxetina Informe de Evaluación. [Comprobado 2101-2013] Disponible en: http://www.navarra.es/home_es/Temas/Portal+de+la+Salud/Profesionales/ 
ORIGINALES Y REVISIONES

Documentacion+y+publicaciones/Publicaciones+tematicas/Medicamento/FET/2008/FET+2008+1. htm

(119) Therapeutics Initiative Atomoxetine for ADHD in children and adolescents University of British Columbia Canada Therapeutics Letter January - March 2009 [Consultado 1-2-2013] Disponible en: http://www.ti.ubc.ca/PDF/73.pdf

(120) Agencia Española de Medicamentos y Productos Sanitarios AEMPS. Atomoxetina (Strattera ${ }^{\circledR}$ ) y aumento de la presión arterial y frecuencia cardíaca: nuevas recomendaciones. 2011. [Consultado 12-12-2012] Disponible en: http://www.aemps.gob.es/informa/notasInformativas/ medicamentosUsoHumano/seguridad/2011/NI-MUH_25-2011.htm

(121) Polzer J, Bangs ME, Zhang S, Dellv MA, Tauscher-Wisniewski S, Acharya N. et al, Meta-Analysis of Aggression or Hostility Events in Randomized, Controlled Clinical Trials of Atomoxetine for ADHD. Biol Psychiatry 2007;61:713-719.

(122) Drug safety update. Atomoxetine: risk of psychotic or manic symptoms. Medicines and Healthcare products Regulatory Agency and Commission on Human Medicines, 8 (2): 4. March 2009. [Consultado 12-12-2012] Disponible en: http://www.mhra.gov.uk/home/groups/pl-p/documents/ publication/con041213.pdf. Comprobado el 19/01/2013

(123) Spealman RD. Noradrenergic involvement in the discriminative stimulus effects of cocaine in squirrel monkeys J Pharmacol Exp Ther. 1995 Oct;275(1):53-62.

(124) Heil SH, Holmes HW, Bickel WK, Higgins ST, Badger GJ, Laws HF, et al. Comparison of the subjective, physiological, and psychomotor effects of atomoxetine and methylphenidate in light drug users. Drug Alcohol Depend. 2002 Jul 1;67(2):149-56.

(125) Bymaster FP, Katner JS, Nelson DL, Hemrick-Luecke SK, Threlkeld PG, Heiligenstein $\mathrm{JH}$, et al. Atomoxetine increases extracellular levels of norepinephrine and dopamine in prefrontal cortex of rat: a potential mechanism for efficacy in attention deficit/hyperactivity disorder. Neuropsychopharmacology. 2002 Nov;27(5):699-711.

(126) Hanwella R, Senanayake M, de Silva V. Comparative efficacy and acceptability of methylphenidate and atomoxetine in treatment of attention deficit hyperactivity disorder in children and adolescents: a meta-analysis. BMC Psychiatry 2011, 11:176. [Consultado 13-1-2013] Disponible en: http://www.biomedcentral.com/1471-244X/11/176

(127) Wang Y, Zheng Y, Du Y, Song DH, Shin YJ, Cho SC, et al. Atomoxetine versus methylphenidate in paediatric outpatients with attention deficit hyperactivity disorder: a randomized, double-blind comparison trial. Aust N Z J Psychiatry. 2007 Mar;41(3):222-30.

(128) Mattai AK, Hill JH, Lenroot RK. Treatment of Early-onset Schizophrenia. Current Opinion in Psychiatry, 2010, 23 (4), 304-310.

(129) NIMH. Press Release July 20, 2007. [Consultado 20-01-2013] Disponible en: http:// www.nimh.nih.gov/science-news/2007/improvement-following-adhd-treatment-sustained-in-mostchildren.shtml

(130) NIMH. Science Update March 26, 2009 [Consultado 20-01-2013] Disponible en: http:// www.nimh.nih.gov/science-news/2009/short-term-intensive-treatment-not-likely-to-improve-longterm-outcomes-for-children-with-adhd.shtml

(131) Santosh PJ, Taylor E, Swanson J, Wigal T, Chuang S, Davies M, et al. Refining the diagnoses of inattention and overactivity syndromes: A reanalysis of the Multimodal Treatment study 
of attention deficit hyperactivity disorder (ADHD) based on ICD10 criteria for hyperkinetic disorder. Clinical and Neuroscience Research, 2005, 5(56), 307314.

(132) Catalá MA, Boluda C, Gutiérrez JG. \& Naenen K. Consentimiento informado en el tratamiento psicofarmacológico de niños y adolescentes: fichas de apoyo al terapeuta. Agencia Valenciana de Salud. Valencia 2008. Ver ficha "Consentimiento informado para el tratamiento con metilfenidato" punto 3. [Consultado el 28-8-2012] Disponible en: http://www.spcv.org/dmdocuments/ Consentimiento_informado_en_ninyos_Fichas_de_apoyo_al_terapeuta.pdf

(133) Prescrire Méthylphénidate : un amphétaminique à ne manier qu'avec beaucoup de précautions. Rev. Prescrire $2011 ; 31$ (330) : 263.

(134) Diller LH. The Last Normal Child: Essays on the Intersection of Kids, Culture, and Psychiatric Drugs. USA: Praeger, 2006.

(135) Stratton A. Ritalin of no long-term benefit, study finds. The Guardian, Monday 12-112007. [Consultado 6-2-2013] Accesible en : http://www.guardian.co.uk/news/2007/nov/12/uknews. health 